\title{
APPROXIMATION OF PLANAR BV HOMEOMORPHISMS BY DIFFEOMORPHISMS
}

\author{
ALDO PRATELLI AND EMANUELA RADICI
}

\begin{abstract}
We show that a planar BV homeomorphism can be approximated in the area strict sense, together with its inverse, with smooth or piecewise affine homeomorphisms.
\end{abstract}

\section{INTRODUCTION}

The problem of approximating homeomorphisms with diffeomorphisms is well-studied and much is known. In particular, in the last years, it was deeply investigated how to approximate Sobolev or bi-Sobolev homeomorphisms in the plane. It is now known that any $W^{1, p}$ homeomorphism defined on some open set $\Omega \subseteq \mathbb{R}^{2}$ can be approximated in the strong $W^{1, p}$ sense by diffeomorphisms (for a proof see $[9,8]$, but also $[3,15]$ ). What remains open is then to consider the case of bi-Sobolev homeomorphisms, that is, Sobolev homeomorphisms whose inverse is also a Sobolev function. Beside its general interest, this question is of primary importance in the study of non-linear elasticity. Up to now, it is only known that an approximation in the strong bi- $W^{1, p}$ sense holds if one considers bi-Lipschitz homeomorphisms (see [5]), or for the special case $p=1$ (see [13]).

In this paper, we give an approximation result for the case of BV homeomorphisms, that is, we show that any BV homeomorphism $u$ in the plane can be approximated by a sequence $\left\{u_{j}\right\}$ of diffeomorphisms (or of piecewise affine homeomorphisms, which is known to be equivalent thanks to [12]). It is important to specify in which sense the sequence converges to the original function: indeed, apart from the case when the BV function is actually in $W^{1,1}$, it is of course impossible to obtain a strong BV convergence, so one has to look for a weak one. There are two notions of weak convergence in BV which are normally used, namely, the weak* convergence and the strict one, which is stronger. We will prove our result by using the "area strict" convergence, introduced in the 1990's by Delladio [6] and recently used by Kristensen, Rindler and Shaw in some papers, see $[10,11,16]$; even if this convergence is less used than the other two weak ones, it is extremely reasonable. In particular, since area strict convergence is even stronger than the strict one, the convergence in particular holds also in both the strict and the weak* sense. The formal definition and a thorough discussion about the area strict convervence are in Section 2, but the idea is very simple; namely, the sequence $u_{j}$ area strict converges to $u$ if it converges strictly, and a part of $D u_{j}$ strongly converges in $L^{1}$ to the absolutely continuous part of $D u$.

Before stating our main result, a couple of comments are useful. First of all, it is known that the inverse of a BV homeomorphism is also BV ([7], see also [4, Theorem 1.3]). And in fact, in our construction not only the sequence $\left\{u_{j}\right\}$ area strict converges to $u$, but also the sequence $\left\{u_{j}^{-1}\right\}$ area strict converges to $u^{-1}$. Hence, our result is somehow in the framework of the open question for bi-Sobolev homeomorphisms.

Moreover, for simplicity, in our result we only claim the existence of a sequence of piecewise affine homeomorphisms which converges to $u$; as said above, this automatically implies also the existence of a converging sequence of diffeomorphisms, thanks to [12]. 
Finally, in our result we will say that each function $u_{j}$ "uniformly coincides with $u$ on $\partial \Omega$ " and is "finitely piecewise affine where possible". The formal definitions of these notions are given in Section 1.1, however they are not really needed to understand the result. Roughly speaking, saying that $u_{j}$ uniformly coincides with $u$ on $\partial \Omega$ simply means that the error $\left|u_{j}-u\right|$ goes to 0 arbitrarily fast when approching the boundary, while saying that $u_{j}$ is finitely piecewise affine where possible means that it is finitely piecewise affine, instead of simply piecewise affine, whenever it makes sense, for instance if $\Omega$ is a polygon and $u$ is piecewise linear on $\partial \Omega$.

We can now state our result.

Theorem A (Area strict approximation in bi-BV). Let $\Omega \subseteq \mathbb{R}^{2}$ be an open set, and let $u: \Omega \rightarrow \Delta$ be a BV homeomorphism. Then, there exists a sequence $\left\{u_{j}\right\}$ of countably piecewise affine $\mathrm{BV}$ homeomorphisms between $\Omega$ and $\Delta$, uniformly coinciding with $u$ on $\partial \Omega$ in the sense of Definition 1.1 and finitely piecewise affine where possible in the sense of Definition 1.2, so that $\left\{u_{j}\right\}$ and $\left\{u_{j}^{-1}\right\}$ converge uniformly and in the area strict sense to $u$ and $u^{-1}$.

The plan of the paper is the following. In Section 1.1 below we list some basic notation that we are going to use; in Section 2 we present and discuss the notion of area strict convergence in BV; in Section 3 we list several results which have been recently proved and that we are going to use; in Section 4 we define the "Lebesgue squares" and we prove their main properties, and finally in Section 5 we present our construction.

1.1. Notation. Here we briefly list the main notation we are going to use through the paper.

Whenever $u$ is a $\operatorname{BV}(\Omega)$ function, we will decompose $D u=\nabla u+D^{s} u$, where $\nabla u$ is the absolutely continuous part of $D u$, while $D^{s} u$ is the singular part. We will use the symbol $\nabla u$ also to denote the density of the measure $\nabla u$ with respect to the Lebesgue measure, thus having $\nabla u \in L^{1}(\Omega)$ : this is a slight abuse of notation, but it simplifies the notation and does not lead to misunderstandings through the paper. Note that, since we work only with BV homeomorphisms, then in particular there is no jump part of $D u$, so $D^{s} u$ is entirely of Cantor type. In particular, $D^{s} u(S)=0$ for every segment $S$.

Whenever $c \in \mathbb{R}^{2}$ and $r>0$, we will denote by $Q(c, r)$ the square with side $2 r$, sides parallel to the coordinate axes, and centered at $c$.

A function $u: \Omega \rightarrow \mathbb{R}^{2}$ is said to be piecewise affine if $\Omega$ can be decomposed as a countable but locally finite union of triangles, on each of which $u$ is affine. The function is said finitely piecewise affine if the above decomposition is a finite one (in particular, $\Omega$ must be a polygon). A Jordan curve $\Gamma: \mathbb{S}^{1} \rightarrow \mathbb{R}^{2}$ is said piecewise linear if $\Gamma$ is a finite union of segments, and given such a curve a function $g: \Gamma \rightarrow \mathbb{R}^{2}$ is said piecewise linear if $\Gamma$ is a finite union of segments on each of which $g$ is linear.

The following two definitions are also used in the paper.

Definition 1.1 (Uniform coincidence at the boundary). Let $\Omega$ be an open set, and fix any continuous, strictly increasing function $\delta \mapsto \eta(\delta)$ with $\eta(0)=0$. Given two homeomorphisms $u, v: \Omega \rightarrow \mathbb{R}^{2}$, we say that $u$ and $v$ uniformly coincide at $\partial \Omega$ if, whenever $x \in \Omega$ has distance less than $\delta$ from $\mathbb{R}^{2} \backslash \Omega$, one has $|u(x)-v(x)|<\eta(\delta)$.

Notice that, if both $u$ and $v$ belong to some Sobolev space $W^{1, p}$, the above definition is stronger than saying that $u-v \in W_{0}^{1, p}$; in particular, not only the traces of $u$ and $v$ coincide on $\partial \Omega$ but also, if both the functions are continuous and one of the two is continuous up to the boundary, then so is also the other one (and the values on the boundary coincide). 
Definition 1.2 (Finitely piecewise affine where possible). Let $u$ be a given BV homeomorphism between two open sets $\Omega$ and $\Delta$ in $\mathbb{R}^{2}$, and let $v$ be another $\mathrm{BV}$ homeomorphism, which uniformly coincides with $u$ on $\partial \Omega$. We say that $v$ is finitely piecewise affine where possible if the following holds. Assume that $\Gamma$ is a piecewise linear Jordan curve, contained in $\partial \Omega$ and with positive distance to $\partial \Omega \backslash \Gamma$, such that $u$ is continuous up to $\Gamma$ and piecewise linear there. Then, there exists a neighborhood of $\Gamma$ on which $v$ is finitely piecewise affine.

Notice that, if $\Omega$ is a polygon and $u$ is finitely piecewise linear on $\partial \Omega$, then a piecewise affine function $v$, uniformly coinciding with $u$ on $\partial \Omega$ and "finitely piecewise affine where possible" is actually finitely piecewise affine.

\section{The AREA STRICT CONVERGENCES}

In this section we recall the well-known definitions of strong, weak* and strict convergence, and then we present and discuss the notion of the area strict convergence. Let $u \in \operatorname{BV}(\Omega)$ be given, and let $\left\{u_{j}\right\}_{j \in \mathbb{N}} \subseteq \mathrm{BV}(\Omega)$ be a sequence which strongly converges to $u$ in $L^{1}(\Omega)$. We say that $u_{j}$ strongly converges to $u$, or simply $u_{j} \rightarrow u$, if the measures $D u_{j}$ strongly converge to $D u$, that is, $\left|D u_{j}-D u\right|(\Omega) \rightarrow 0$. This is the standard convergence related to the BV norm. We say that $u_{j}$ weakly* converges to $u$, or $u_{j}{ }^{*} u$, if the measures $D u_{j}$ weakly* converge to $D u$ with respect to the duality with the continuous and bounded functions, that is, for every continuous and bounded function $\phi: \Omega \rightarrow \mathbb{R}$ one has $\int_{\Omega} \phi d D u_{j} \rightarrow \int_{\Omega} \phi d D u$. We say that $u_{j}$ strict converges to $u$ if it weakly* converges, and in addition the total variations $\left|D u_{j}\right|(\Omega)$ converge to the total variation $|D u|(\Omega)$.

In order to introduce the area strict convergence, it is useful to keep in mind that it is always important to have density of the smooth functions. However, smooth functions are clearly not strongly dense in $\operatorname{BV}(\Omega)$ : indeed, if $v$ is any smooth function, then for sure $|D v-D u|(\Omega) \geq$ $\left|D^{s} u\right|(\Omega)$, so the density is obviously false unless $u$ is actually $W^{1,1}$. This is one of the main reasons why one uses the weak* or the strict convergence, since in fact smooth functions are dense in $\mathrm{BV}$ in the strict sense. However, notice that if $u_{j}$ is a sequence of smooth functions, the norm convergence of $D u_{j}$ to $D u$ is only prevented by the presence of the singular part $D^{s} u$, while $\nabla u$ is an $L^{1}$ function, so it can clearly be an $L^{1}$ limit of smooth functions. This motivates us to give the following definition.

Definition 2.1 (Area strict convergence). Let $\Omega \subseteq \mathbb{R}^{2}$ be an open set, let $u \in \mathrm{BV}\left(\Omega, \mathbb{R}^{2}\right)$, and let $\left\{u_{j}\right\} \in \operatorname{BV}\left(\Omega, \mathbb{R}^{2}\right)$ be a sequence converging to $u$ in $L^{1}(\Omega)$. We say that $\left\{u_{j}\right\}$ converges in the area strict sense to $u$, or $u_{j} \stackrel{\text { area }}{\longrightarrow} u$, if it is possible to decompose $D u_{j}=\mu_{j}+\nu_{j}$ with $\left|\mu_{j}-\nabla u\right|(\Omega) \rightarrow 0$ and $\left|\nu_{j}\right|(\Omega) \rightarrow\left|D^{s} u\right|(\Omega)$.

It is clear that this convergence is weaker than the strong one, but stronger than the strict one, and simple examples show that it does not coincide with neither of the two. This notion of convergence was introduced by Delladio in [6], and recently used in $[10,11,16]$, who are only concerned with the case of a bounded domain $\Omega$; their definition is actually quite different, at a first glance: more precisely, for every function $u \in \mathrm{BV}(\Omega)$ and a set $A \subseteq \Omega$ they introduce the functional

$$
\mathcal{F}(u, A):=\int_{A} \sqrt{1+|\nabla u(x)|^{2}} d x+\left|D^{s} u\right|(A),
$$

and they say that $u_{j}$ area strict converges to $u$ if $u_{j}$ strongly converges to $u$ in $L^{1}$ and $\mathcal{F}\left(u_{j}, \Omega\right) \rightarrow$ $\mathcal{F}(u, \Omega)$. Observe that this definition perfectly justifies the use of the word "area". It is clear that 
this approach is meaningful only if $\Omega$ has finite measure, since otherwise $\mathcal{F}(u, \Omega)$ is constantly $+\infty$; on the other hand, for sets of finite measure our definition is equivalent to their one, as we show in a moment in Lemma 2.3 below. An interesting link between $L^{1}$ convergence and convergence of functionals of the above form is also in the paper [2]. Observe that, since $t \leq \sqrt{1+t^{2}} \leq t+1$ for every $t$, then for any two BV functions $u$ and $v$ on $\Omega$, and for any $A \subseteq \Omega$, we have

$$
|D u|(A) \leq \mathcal{F}(u, A) \leq|D u|(A)+|A|, \quad|D v|(A) \leq \mathcal{F}(v, A) \leq|D v|(A)+|A|,
$$

and as a consequence

$$
|\mathcal{F}(u, A)-\mathcal{F}(v, A)| \leq|| D u|(A)-| D v|(A)|+|A| \quad \forall A \subseteq \Omega .
$$

A standard property, which we prove just for the sake of completeness, is the following.

Lemma 2.2. Let $\left\{f_{j}\right\} \in L^{1}(\Omega)$ be a sequence of positive functions which weakly* converges to $f \in L^{1}(\Omega)$, with some set $\Omega$ of finite measure. Then

$$
\int_{\Omega} \sqrt{1+f^{2}} \leq \liminf \int_{\Omega} \sqrt{1+f_{j}^{2}}
$$

and, if $\int_{\Omega} \sqrt{1+f_{j}^{2}} \rightarrow \int_{\Omega} \sqrt{1+f^{2}}$, then $f_{j} \rightarrow f$ strongly in $L^{1}(\Omega)$.

Proof. Since $t \mapsto \sqrt{1+t^{2}}$ is convex, for every $x, y \in \mathbb{R}$ we have

$$
\sqrt{1+(x+y)^{2}} \geq \sqrt{1+x^{2}}+y \frac{x}{\sqrt{1+x^{2}}}+y^{2} \frac{1}{2\left(1+x^{2}\right)^{3 / 2}} \geq \sqrt{1+x^{2}}+y \frac{x}{\sqrt{1+x^{2}}} .
$$

As a first consequence, we have

$$
\int_{\Omega} \sqrt{1+f_{j}^{2}} \geq \int_{\Omega} \sqrt{1+f^{2}}+\int_{\Omega}\left(f_{j}-f\right) \frac{f}{\sqrt{1+f^{2}}}
$$

and, since the latter integral converges to 0 because $f_{j}{ }^{*} f$ in $L^{1}$ while $f /\left(1+f^{2}\right)^{1 / 2} \in L^{\infty}$, we deduce (2.2).

Suppose now that $\int_{\Omega} \sqrt{1+f_{j}^{2}} \rightarrow \int_{\Omega} \sqrt{1+f^{2}}$. Let $\varepsilon>0$ be fixed, and let $M \gg 1$ be such that, calling $A_{M}=\{f>M\}$, one has $\|f\|_{L^{1}\left(A_{M}\right)}+\left|A_{M}\right| / 2 M<\varepsilon$. We have then

$$
\int_{\Omega} \sqrt{1+f_{j}^{2}} \geq \int_{\Omega} \sqrt{1+f^{2}}+\int_{\Omega}\left(f_{j}-f\right) \frac{f}{\sqrt{1+f^{2}}}+\int_{\Omega \backslash A_{M}}\left(f_{j}-f\right)^{2} \frac{1}{2\left(1+f^{2}\right)^{3 / 2}},
$$

and the assumption that $\int_{\Omega} \sqrt{1+f_{j}^{2}} \rightarrow \int_{\Omega} \sqrt{1+f^{2}}$, together with the fact that $\left(1+f^{2}\right)^{-3 / 2}$ is bounded from below in $\Omega \backslash A_{M}$, implies that $f_{j}-f$ strongly converges to 0 in $L^{2}\left(\Omega \backslash A_{M}\right)$, so in particular $f_{j} \rightarrow f$ in $L^{1}\left(\Omega \backslash A_{M}\right)$. On the other hand, we can also estimate

$$
\begin{aligned}
\int_{\Omega} \sqrt{1+f_{j}^{2}} & \geq \int_{\Omega \backslash A_{M}} \sqrt{1+f^{2}}+\int_{\Omega \backslash A_{M}}\left(f_{j}-f\right) \frac{f}{\sqrt{1+f^{2}}}+\left\|f_{j}\right\|_{L^{1}\left(A_{M}\right)} \\
& \geq \int_{\Omega} \sqrt{1+f^{2}}+\int_{\Omega \backslash A_{M}}\left(f_{j}-f\right) \frac{f}{\sqrt{1+f^{2}}}+\left\|f_{j}\right\|_{L^{1}\left(A_{M}\right)}-\|f\|_{L^{1}\left(A_{M}\right)}-\frac{1}{2 M}\left|A_{M}\right| .
\end{aligned}
$$

Since the last integral converges to 0 because the restriction of $f /\left(1+f^{2}\right)^{1 / 2}$ to $\Omega \backslash A_{M}$ is still a $L^{\infty}$ function, we obtain

$$
\limsup \left\|f_{j}\right\|_{L^{1}\left(A_{M}\right)} \leq\|f\|_{L^{1}\left(A_{M}\right)}+\frac{1}{2 M}\left|A_{M}\right|<\varepsilon .
$$

Together with the fact that $f_{j} \rightarrow f$ strongly in $L^{1}\left(\Omega \backslash A_{M}\right)$, this gives lim sup $\left\|f_{j}-f\right\|_{L^{1}(\Omega)}<2 \varepsilon$. Since $\varepsilon$ was arbitrary, we have concluded. 
Lemma 2.3. Let $\Omega$ be a set of finite measure, and let $u_{j} \in \mathrm{BV}(\Omega)$ be a sequence which converges in the $L^{1}$ sense to $u \in \operatorname{BV}(\Omega)$. Then $\mathcal{F}(u, \Omega) \leq \liminf \mathcal{F}\left(u_{j}, \Omega\right)$, and $u_{j}$ converges in the area strict sense to $u$ if and only if $\mathcal{F}\left(u_{j}, \Omega\right) \rightarrow \mathcal{F}(u, \Omega)$.

Proof. We divide the proof in three short steps.

Step I. The area strict convergence implies the convergence of the functional.

First of all, let us assume that $u_{j} \stackrel{\text { area }}{\longrightarrow} u$ and let $\mu_{j}$ and $\nu_{j}$ be as in Definition 2.1. Then, let $\varepsilon>0$ be given, and let $\delta \leq \varepsilon$ be such that $\|\nabla u\|_{L^{1}(B)}<\varepsilon$ for every set $B$ with measure $|B|<\delta$. Let then $A_{\delta}$ be an open set on which $D^{s} u$ is concentrated, with $\left|A_{\delta}\right|<\delta$, so that

$$
\|\nabla u\|_{L^{1}\left(A_{\delta}\right)}<\varepsilon, \quad\left|\mu_{j}\right|\left(A_{\delta}\right)<2 \varepsilon,
$$

where the second inequality holds for every $j \gg 1$, since $\mu_{j} \rightarrow \nabla u$ in the sense of measures. Since $D u_{j}$ is bounded in measure and $u_{j} \stackrel{L^{1}}{\rightarrow} u$, we have that $u_{j} \stackrel{*}{\rightarrow} u$, hence $\nu_{j} \stackrel{*}{\rightarrow} D^{s} u$ in the sense of measures. Thus, being $\Omega \backslash A_{\delta}$ closed, we have

$$
0=\left|D^{s} u\right|\left(\Omega \backslash A_{\delta}\right) \geq \limsup \left|\nu_{j}\right|\left(\Omega \backslash A_{\delta}\right)
$$

from which we obtain

$$
\left|\nu_{j}\right|\left(\Omega \backslash A_{\delta}\right)<\varepsilon
$$

for $j \gg 1$. Then

$$
\left|D u_{j}-D u\right|\left(\Omega \backslash A_{\delta}\right) \leq\left|\mu_{j}-\nabla u\right|\left(\Omega \backslash A_{\delta}\right)+\left|\nu_{j}\right|\left(\Omega \backslash A_{\delta}\right)<2 \varepsilon
$$

as soon as $j \gg 1$, again keeping in mind that $\mu_{j} \rightarrow \nabla u$ in the sense of measures. Recalling that $t \mapsto \sqrt{1+t^{2}}$ is 1-Lipschitz, for $j \gg 1$ and by (2.1), (2.5), (2.3) and (2.4), we can then evaluate

$$
\begin{aligned}
\left|\mathcal{F}(u, \Omega)-\mathcal{F}\left(u_{j}, \Omega\right)\right| & \leq\left|\mathcal{F}\left(u, \Omega \backslash A_{\delta}\right)-\mathcal{F}\left(u_{j}, \Omega \backslash A_{\delta}\right)\right|+\left|\mathcal{F}\left(u, A_{\delta}\right)-\mathcal{F}\left(u_{j}, A_{\delta}\right)\right| \\
& \leq\left|D u_{j}-D u\right|\left(\Omega \backslash A_{\delta}\right)+|| D u\left|\left(A_{\delta}\right)-\right| D u_{j}\left|\left(A_{\delta}\right)\right|+\left|A_{\delta}\right| \\
& \leq 3 \varepsilon+|| D^{s} u|(\Omega)-| \nu_{j}|(\Omega)|+|| \nabla u \|_{L^{1}\left(A_{\delta}\right)}+\left|\mu_{j}\right|\left(A_{\delta}\right)+\left|\nu_{j}\right|\left(\Omega \backslash A_{\delta}\right) \\
& \leq 7 \varepsilon+|| D^{s} u|(\Omega)-| \nu_{j}|(\Omega)| \leq 8 \varepsilon,
\end{aligned}
$$

where the last inequality holds for $j \gg 1$ by area strict convergence. Hence, $\mathcal{F}\left(u_{j}, \Omega\right) \rightarrow \mathcal{F}(u, \Omega)$.

Step II. Lower semicontinuity of $\mathcal{F}$.

Let us show that $\mathcal{F}(u, \Omega) \leq \liminf \mathcal{F}\left(u_{j}, \Omega\right)$. There is nothing to prove if the liminf is $+\infty$, so we can assume without loss of generality, and up to a subsequence, that $\mathcal{F}\left(u_{j}, \Omega\right)$ is bounded, hence that the measures $D u_{j}$ are bounded. Let for a moment $\varepsilon>0$ be fixed, and let $C \subset \subset \Omega$ be a compact, negligible set such that $\left|D^{s} u\right|(\Omega \backslash C)<\varepsilon$. Let also $\delta=\delta(\varepsilon)<\varepsilon$ be chosen in such a way that the open set $A_{\varepsilon}=\{x \in \Omega$ : $\operatorname{dist}(x, C)<\delta\}$ satisfies

$$
|D u|\left(\partial A_{\varepsilon}\right)=\left|D u_{j}\right|\left(\partial A_{\varepsilon}\right)=0 \quad \forall j \in \mathbb{N}, \quad\left|A_{\varepsilon}\right|<\varepsilon, \quad\|\nabla u\|_{L^{1}\left(A_{\varepsilon}\right)}<\varepsilon .
$$

Let us then call $\mu_{j}^{\varepsilon}=D u_{j}\left\llcorner\left(\Omega \backslash A_{\varepsilon}\right)\right.$ and $\nu_{j}^{\varepsilon}=D u_{j}\left\llcorner A_{\varepsilon}=D u_{j}-\mu_{j}^{\varepsilon}\right.$. Since the sequences $\mu_{j}^{\varepsilon}$ and $\nu_{j}^{\varepsilon}$ are bounded, up to a subsequence we have that $\mu_{j}^{\varepsilon}{ }^{*} \mu^{\varepsilon}$ and $\nu_{j}^{\varepsilon}{ }^{*} \nu^{\varepsilon}$ with $\mu^{\varepsilon}+\nu^{\varepsilon}=D u$. Since $A_{\varepsilon}$ and $\Omega \backslash \overline{A_{\varepsilon}}$ are open sets, one has

$$
\left|\mu^{\varepsilon}\right|\left(A_{\varepsilon}\right) \leq \liminf \left|\mu_{j}^{\varepsilon}\right|\left(A_{\varepsilon}\right)=0, \quad\left|\nu^{\varepsilon}\right|\left(\Omega \backslash \overline{A_{\varepsilon}}\right) \leq \liminf \left|\nu_{j}^{\varepsilon}\right|\left(\Omega \backslash \overline{A_{\varepsilon}}\right)=0 .
$$


Let us now call $H_{\xi}=\{x \in \Omega: \delta-\xi<\operatorname{dist}(x, C)<\delta+\xi\}$. Since $H_{\xi}$ is open, as well as $\Omega \backslash \overline{H_{\xi}}$, we have

$$
\left|\mu^{\varepsilon}\right|(\partial C) \leq\left|\mu^{\varepsilon}\right|\left(H_{\xi}\right) \leq \liminf _{j \rightarrow \infty}\left|D u_{j}\right|\left(H_{\xi} \backslash A_{\varepsilon}\right) \leq \limsup _{j \rightarrow \infty}\left|D u_{j}\right|\left(\overline{H_{\xi}}\right) \leq|D u|\left(\overline{H_{\xi}}\right),
$$

and since the latter goes to 0 for $\xi \searrow 0$ we deduce that $\mu^{\varepsilon}(\partial C)=0$. From (2.7) and (2.6) we derive then

$$
\mu^{\varepsilon}=D u\left\llcorner\left(\Omega \backslash A_{\varepsilon}\right), \quad \nu^{\varepsilon}=D u\left\llcorner A_{\varepsilon} .\right.\right.
$$

Notice that, by $(2.6)$ and since $\left|D^{s} u\right|\left(\Omega \backslash A_{\varepsilon}\right)<\varepsilon$, if $\varepsilon \searrow 0$, then $\mu^{\varepsilon}$ and $\nu^{\varepsilon}$ strongly converge to $\nabla u$ and $D^{s} u$ respectively. As a consequence, with a standard triangular argument, we can call $\mu_{j}^{\prime}=\mu_{j}^{\varepsilon_{j}}$ and $\nu_{j}^{\prime}=\nu_{j}^{\varepsilon_{j}}$ for a suitable sequence $\varepsilon_{j}$, going to 0 slowly enough so that

$$
\mu_{j}^{\prime} \stackrel{*}{\longrightarrow} \nabla u, \quad \nu_{j}^{\prime} \stackrel{*}{\longrightarrow} D^{s} u .
$$

Finally, let us call $\alpha_{j}$ the singular part of $\mu_{j}^{\prime}$ and let

$$
\mu_{j}=\mu_{j}^{\prime}-\alpha_{j}, \quad \nu_{j}=\nu_{j}^{\prime}+\alpha_{j} .
$$

Notice that $\alpha_{j}$ strongly converges to 0 , so also $\mu_{j}$ and $\nu_{j}$ weak* converge to $\nabla u$ and $D^{s} u$. However, $\mu_{j} \in L^{1}(\Omega)$. Observe that

$$
\begin{aligned}
\mathcal{F}\left(u_{j}, \Omega\right) & =\mathcal{F}\left(u_{j}, \Omega \backslash A_{\varepsilon_{j}}\right)+\mathcal{F}\left(u_{j}, A_{\varepsilon_{j}}\right)=\int_{\Omega \backslash A_{\varepsilon_{j}}} \sqrt{1+\left|\mu_{j}\right|^{2}}+\left|\alpha_{j}\right|(\Omega)+\mathcal{F}\left(u_{j}, A_{\varepsilon_{j}}\right) \\
& \geq \int_{\Omega \backslash A_{\varepsilon_{j}}} \sqrt{1+\left|\mu_{j}\right|^{2}}+\left|\alpha_{j}\right|(\Omega)+\left|\nu_{j}^{\prime}\right|(\Omega)=\int_{\Omega} \sqrt{1+\left|\mu_{j}\right|^{2}}+\left|\nu_{j}\right|(\Omega)-\left|A_{\varepsilon_{j}}\right|
\end{aligned}
$$

By Lemma 2.2 we can then evaluate

$$
\begin{aligned}
\mathcal{F}(u, \Omega) & =\int_{\Omega} \sqrt{1+|\nabla u|^{2}}+\left|D^{s} u\right|(\Omega) \leq \liminf \int_{\Omega} \sqrt{1+\left|\mu_{j}\right|^{2}}+\liminf \left|\nu_{j}\right|(\Omega) \\
& \leq \liminf \int_{\Omega} \sqrt{1+\left|\mu_{j}\right|^{2}}+\left|\nu_{j}\right|(\Omega) \leq \liminf \mathcal{F}\left(u_{j}, \Omega\right) .
\end{aligned}
$$

The lower semicontinuity of $\mathcal{F}$ is then obtained.

Step III. The convergence of the functional implies the area strict convergence.

To conclude, let us assume that $\mathcal{F}\left(u_{j}, \Omega\right) \rightarrow \mathcal{F}(u, \Omega)$. We define the sequences $\left\{\mu_{j}\right\}$ and $\left\{\nu_{j}\right\}$ as in Step II, so that the inequality (2.8) holds true, and it must actually be an equality. This implies at once that $\left|\nu_{j}\right|(\Omega) \rightarrow\left|D^{s} u\right|(\Omega)$, and that $\int_{\Omega} \sqrt{1+\left|\mu_{j}\right|^{2}} \rightarrow \int_{\Omega} \sqrt{1+|\nabla u|^{2}}$, which in turn gives $\mu_{j} \rightarrow \nabla u$ strongly in $L^{1}(\Omega)$ by Lemma 2.2 .

We conclude this section with a known technical result, which represents a link between the directional derivatives of a $\mathrm{BV}$ function and the derivatives of the one-dimensional sections, and with a final remark.

Lemma 2.4 ([1], Theorem 3.103). Let $Q$ be a square, and let $u \in \mathrm{BV}(Q)$. Then, for almost every $y$ the function $u_{y}(x)=u(x, y)$ is a BV one-dimensional function, and moreover $D_{1} u=D u_{y} d y$, where $D u_{y}$ is the derivative of the BV function $u_{y}$.

Remark 2.5. Let us notice that, in the proof of the Lemma 2.3, we did not really use the fact that the integrand is precisely $\sqrt{1+t^{2}}$, but only that it is a strictly convex function linear at 
infinity (so, in particular, Lipschitz). Therefore, the very same proof actually shows that the area strict convergence on sets with finite measure is equivalent to the convergence of the functional

$$
\mathcal{F}_{\phi}(v, A):=\int_{A} \phi(|\nabla v(x)|) d x+\phi_{\infty}\left|D^{s} v\right|(A) \quad \forall v \in \mathrm{BV}(\Omega), A \subseteq \Omega
$$

for any positive, strictly convex function $\phi$ with recession $\phi_{\infty}=\lim _{t \rightarrow \infty} \phi(t) / t<\infty$.

\section{Preliminaries}

This section is devoted to present several known results, which have been proved in the last few years, and which we will need later. We start with two geometrical facts taken from [8]: the first one is a simplified version of [8, Proposition 4.18], and the second one is [8, Theorem 3.1].

Lemma 3.1. Let $\Gamma=\cup_{j=1}^{P} \mathcal{S}_{j}$ be a finite union of segments in the interior of $\Omega$, and let $\Gamma_{0}$ be a collection of some of these segments. Moreover, let $\xi>0$ be a fixed constant, and $g: \Gamma \rightarrow \mathbb{R}^{2}$ be a given injective function. Then, inside each segment $\mathcal{S}_{j}$ there are finitely many essentially disjoint subsegments $\mathcal{S}_{j}^{i}$, each of which with length at most $\xi$, such that the following holds. Every segment $\mathcal{S}_{j}^{i}$ lies entirely within a distance $\xi$ from $\Gamma_{0}$, so in particular if $\mathcal{S}_{j}$ has distance larger than $\xi$ from $\Gamma_{0}$ there is no segment $\mathcal{S}_{j}^{i}$; on the other hand, if $\mathcal{S}_{j} \in \Gamma_{0}$ then the union of the segments $\mathcal{S}_{j}^{i}$ is the whole $\mathcal{S}_{j}$. Moreover, defining $\varphi: \Gamma \rightarrow \mathbb{R}^{2}$ the function which is linear on each segment $\mathcal{S}_{j}^{i}$, coinciding with $g$ on the two endpoints of $\mathcal{S}_{j}^{i}$, and which coincides with $g$ outside the union of the segments $\mathcal{S}_{i}^{j}$, we have that $\varphi$ is still injective.

Theorem 3.2. There exist two purely geometric positive constants $\bar{\varepsilon}$ and $K$ such that, if $Q$ is a square of side $2 r, \varphi: \partial Q \rightarrow \mathbb{R}^{2}$ is a piecewise linear and injective function, $M$ is a matrix with $\operatorname{det} M=0$, and

$$
|D \varphi-M \cdot \tau|(\partial Q) \leq r\|M\| \bar{\varepsilon},
$$

where $\tau$ denotes the tangent direction on $\partial Q$, then there exists a finitely piecewise affine extension $v: Q \rightarrow \mathbb{R}^{2}$ of $\varphi$ such that

$$
\int_{Q}|D v-M| \leq K r|D \varphi-M \cdot \tau|(\partial Q) .
$$

Let us now recall some useful results about planar homeomorphisms of bounded variations. The first one generalizes to the BV setting the simple identity $\|D u\|_{L^{1}(\Omega)}=\left\|D u^{-1}\right\|_{L^{1}(u(\Omega))}$ that holds for planar bi-Sobolev homeomorphisms, and it was proved in [4, Theorem 1.3].

Theorem 3.3. Let $\Omega, \Delta \subseteq \mathbb{R}^{2}$ be open and suppose that $u: \Omega \rightarrow \Delta$ is a homeomorphism. Then $u \in \operatorname{BV}\left(\Omega, \mathbb{R}^{2}\right)$ if and only if $u^{-1} \in \operatorname{BV}\left(\Delta, \mathbb{R}^{2}\right)$. Moreover, for every $A \subseteq \Omega$ one has

$$
|D u|(A)=\left|D u^{-1}\right|(u(A)) .
$$

We present now some results contained in the paper [14], which is appearing contemporarely to this one. Let $\mathcal{R}$ be a rectangle with the sides parallel to the coordinate axes, and let $\partial \mathcal{R}$ be its boundary. For every Jordan curve $\varphi: \partial \mathcal{R} \rightarrow \mathbb{R}^{2}$, we will call $\mathcal{P}=\mathcal{P}(\varphi)$ the internal part of this curve, that is, the closed, bounded, connected component of $\mathbb{R}^{2} \backslash \varphi(\partial \mathcal{R})$; since we will almost always consider piecewise linear maps $\varphi$, the sets $\mathcal{P}$ will usually be polygons. We define the "minimal energy of $\varphi$ " as follows.

Definition 3.4. Let $\mathcal{R}=\left[a^{-}, a^{+}\right] \times\left[b^{-}, b^{+}\right]$be a rectangle, and let $\varphi: \partial \mathcal{R} \rightarrow \mathbb{R}^{2}$ and $\mathcal{P}$ be $a$ Jordan curve and its internal part. For every $x, y \in \mathcal{P}$, we call $d_{\mathcal{P}}(x, y)$ the geodesic distance 
in $\mathcal{P}$ between $x$ and $y$, that is, the length of the shortest path connecting $x$ and $y$ inside $\mathcal{P}$. The minimal energy of $\varphi$ is the number

$$
\Psi(\varphi)=\int_{a^{-}}^{a^{+}} d_{\mathcal{P}}\left(\varphi\left(t, b^{-}\right), \varphi\left(t, b^{+}\right)\right) d t+\int_{b^{-}}^{b^{+}} d_{\mathcal{P}}\left(\varphi\left(a^{-}, t\right), \varphi\left(a^{+}, t\right)\right) d t .
$$

The reason for the name comes from the following simple result (actually much more is true, namely, the "minimal energy" is really the minimal one, without multiplicative constants, as soon as one considers the Manhattan distance instead of the Euclidean one, see [14, Theorem A]).

Lemma 3.5. Let $u: \Omega \rightarrow \Delta$ be a BV homeomorphism, let $\mathcal{R} \subset \subset \Omega$ be a rectangle, and let $\varphi$ be the restriction of $u$ to $\partial \mathcal{R}$. Then $|D u|(\mathcal{R}) \geq \frac{\sqrt{2}}{2} \Psi(\varphi)$.

Proof. For simplicity of notation, we assume that $\mathcal{R}=[0,1]^{2}$ is the unit square. Keep in mind that, if $\mathcal{S} \subseteq \mathbb{R}^{2}$ is a segment and $\psi: \mathcal{S} \rightarrow \mathbb{R}^{2}$ is a continuous BV curve, then the total variation $|D \psi|(\mathcal{S})$ is greater than or equal to the length of the curve; in particular, by Lemma 2.4, for almost every $0 \leq t \leq 1$, if we call $\mathcal{S}_{t}$ the horizontal segment $\{(s, t), 0 \leq s \leq 1\}$ and $\psi$ the restriction of $u$ to $\mathcal{S}_{t}$, we get

$$
\left|D_{1} u\right|\left(S_{t}\right)=|D \psi|\left(\mathcal{S}_{t}\right) \geq d_{\mathcal{P}}(\varphi(0, t), \varphi(1, t)),
$$

where the last inequality is due to the fact that $\psi$ is a curve contained in $\mathcal{P}(\varphi)$ and connecting $\varphi(0, t)$ with $\varphi(1, t)$. Similarly, calling $\mathcal{S}^{t}$ the vertical segment $\{(t, s), 0 \leq s \leq 1\}$, we have

$$
\left|D_{2} u\right|\left(\mathcal{S}^{t}\right) \geq d_{\mathcal{P}}(\varphi(t, 0), \varphi(t, 1)) .
$$

Finally, just by integrating, directly by Definition 3.4 we get

$$
\sqrt{2}|D u|(\mathcal{R}) \geq\left|D_{1} u\right|(\mathcal{R})+\left|D_{2} u\right|(\mathcal{R})=\int_{t=0}^{1}\left|D_{1} u\right|\left(\mathcal{S}_{t}\right)+\int_{t=0}^{1}\left|D_{2} u\right|\left(\mathcal{S}^{t}\right) \geq \Psi(\varphi),
$$

which concludes the proof.

We now see how the function $\Psi$ varies if we slightly modify the boundary value $\varphi$. More precisely, we will consider the following variations of a boundary value.

Definition 3.6 ( $\eta$-linearization of a Jordan curve). Let $g: \partial \mathcal{R} \rightarrow \mathbb{R}^{2}$ be a given Jordan curve, let $\mathcal{S}_{1}, \mathcal{S}_{2}, \ldots, \mathcal{S}_{P}$ be finitely many essentially disjoint segments in $\partial \mathcal{R}$. We define the continuous curve $\varphi: \partial \mathcal{R} \rightarrow \mathbb{R}^{2}$ as the function which coincides with $g$ on $\partial \mathcal{R} \backslash \bigcup_{i=1}^{P} \mathcal{S}_{i}$, and which is the segment connecting the images under $g$ of the two endpoints with constant speed in each $\mathcal{S}_{i}$. We say that $\varphi$ is an $\eta$-linearization of $g$, for $\eta>0$ much smaller than the diameter of $g(\partial \mathcal{R})$, if $\varphi$ is injective and, for every $1 \leq i \leq P$, the curve $g$ on $\mathcal{S}_{i}$ has length smaller than $\eta$ and intersects $\varphi$ only within the segment $\varphi\left(\mathcal{S}_{i}\right)$ (but not necessarily only at the endpoints of this segment).

The next three results are taken from [14], in particular the first one is [14, Corollary 4.3], the second is an obvious consequence of [14, Theorem A], and the third one is [14, Theorem 1.4 and Remark 4.4]. Notice that, also by Theorem 3.3, the last result is exactly the same as our Theorem A, except that the area strict convergence is replaced by the (weaker) strict convergence.

Lemma 3.7. Let $g: \partial \mathcal{R} \rightarrow \mathbb{R}^{2}$ be a Jordan curve and let $\varphi$ be an $\eta$-linearization. Then

$$
\Psi(\varphi) \leq \Psi(g)+\eta \mathscr{H}^{1}(\partial \mathcal{R}) .
$$


Proposition 3.8. Let $\mathcal{R}$ be a rectangle, and let $\varphi: \partial \mathcal{R} \rightarrow \mathbb{R}^{2}$ be a continuous injective function. Then, there exists a piecewise affine homeomorphism $v: \mathcal{R} \rightarrow \mathbb{R}^{2}$ such that $v=\varphi$ on $\partial \mathcal{R}$ and

$$
\int_{\mathcal{R}}|D v| \leq \Psi(\varphi)
$$

Moreover, if $\varphi$ is piecewise linear then the function $v$ can be taken finitely piecewise affine.

Theorem 3.9. Let $\Omega \subseteq \mathbb{R}^{2}$ be an open set, and let $u: \Omega \rightarrow \Delta$ be a BV homeomorphism. Then, there exists a sequence $\left\{u_{j}\right\}$ of countably piecewise affine BV homeomorphisms between $\Omega$ and $\Delta$, uniformly coinciding with $u$ on $\partial \Omega$ in the sense of Definition 1.1 and finitely piecewise affine where possible in the sense of Definition 1.2, such that $\left\{u_{j}\right\}$ and $\left\{u_{j}^{-1}\right\}$ converge uniformly and strictly to $u$ and $u^{-1}$.

\section{Definition And properties of the Lebesgue squares}

This section is devoted to the definition and the study of the "Lebesgue squares", which are, roughly speaking, squares inside the domain where the function is sufficiently close to an affine mapping. The same concept has been already successfully used several times in the last years (see for instance $[5,13,8]$ ), but always in the framework of Sobolev or bi-Sobolev functions. As a consequence, we need now to give a more general definition, to cover also the case of BV homeomorphisms.

Definition 4.1 (Lebesgue squares). Let $u \in \operatorname{BV}\left(\Omega, \mathbb{R}^{2}\right)$ be a homeomorphism, and let $c \in \Omega$ and $r>0$ be such that $Q(c, 3 r) \subset \subset \Omega$. We say that $Q(c, r)$ is a Lebesgue square corresponding to the matrix $M \in \mathbb{R}^{2 \times 2}$ and to the constant $\delta \leq 1$ if

$$
|D u-M|(Q(c, 3 r))<r^{2} \delta .
$$

The main utility of the Lebesgue squares comes from the following simple fact, which ensures that in such a square the function $u$ is uniformly close to an affine function $\omega$ with $D \omega=M$. The very same result has been already noticed and used several times for Sobolev functions, see for instance [8, Lemma 4.3]; the proof in the more general framework of BV functions is also very similar, one only has to be careful that $D u$ is a measure instead of an $L^{1}$ function.

Lemma 4.2. Let $Q=Q(c, r)$ be a Lebesgue square with matrix $M$ and constant $\delta$. For every $\varepsilon>0$ there exists $\bar{\delta}=\bar{\delta}(\varepsilon,\|M\|) \ll \varepsilon$ such that if $\delta \leq \bar{\delta}$, then

$$
\|u-\omega\|_{L^{\infty}(Q(c, 2 r))} \leq \varepsilon r
$$

where $\omega: \mathbb{R}^{2} \rightarrow \mathbb{R}^{2}$ is a suitable affine function satisfying $D \omega=M$. If in addition $\operatorname{det} M>0$, then, provided $\delta \leq \bar{\delta}^{\prime}(\varepsilon,\|M\|$, $\operatorname{det} M)$,

$$
\omega(Q(c,(1-\varepsilon) r)) \subseteq u\left(Q\left(c,\left(1-\frac{2}{3} \varepsilon\right) r\right)\right) \subseteq w\left(Q\left(c,\left(1-\frac{\varepsilon}{3}\right) r\right)\right) \subseteq u(Q) \subseteq \omega(Q(c,(1+\varepsilon) r)) .
$$

Proof. Without loss of generality, we can assume that $Q$ is centered at the origin. By Lemma 2.4 we decompose $D_{1} u=D u_{y} d y$ and $D_{2} u=D u^{x} d x$, where, for almost every $x, y, D u_{y}$ and $D u^{x}$ are the derivatives of the BV functions $u_{y}$ and $u^{x}$, being $u_{y}(t)=u(t, y)$ and $u^{x}(t)=u(x, t)$.

Let then $R$ be a large constant, to be specified later: we define

$$
\begin{aligned}
& A=\left\{x \in(-3 r, 3 r):\left|D u^{x}-M_{2}\right|(-3 r, 3 r) \leq R \delta r\right\}, \\
& B=\left\{y \in(-3 r, 3 r):\left|D u_{y}-M_{1}\right|(-3 r, 3 r) \leq R \delta r\right\},
\end{aligned}
$$


where $M_{1}$ and $M_{2}$ are the two rows of the matrix $M$, and we notice that by the definition of Lebesgue square we have

$$
|(-3 r, 3 r) \backslash A|<\frac{r}{R}, \quad \quad|(-3 r, 3 r) \backslash B|<\frac{r}{R},
$$

Let us now select a point $a=\left(a_{1}, a_{2}\right)$ such that $a_{1} \in A, a_{2} \in B$, and let us define the auxiliary BV functions $\omega(z)=M(z-a)+u(a)$, and $\psi(z)=u(z)-\omega(z)$. For every $x \in A, y \in(-3 r, 3 r)$, then, since $\psi(a)=0$ we have

$$
\begin{aligned}
|\psi(x, y)| & \leq\left|\psi(x, y)-\psi\left(x, a_{2}\right)\right|+\left|\psi\left(x, a_{2}\right)-\psi\left(a_{1}, a_{2}\right)\right| \\
& \leq\left|D u^{x}-M_{2}\right|\left(a_{2}, y\right)+\left|D u_{a_{2}}-M_{1}\right|\left(x, a_{1}\right) \leq 2 R \delta r
\end{aligned}
$$

and the obvious modification of the argument implies that the estimate holds true also for $x \in(-3 r, 3 r), y \in B$.

Let now $(x, y)$ be any point in $Q(c, 2 r)$; thanks to (4.3) we can select $x^{-}<x<x^{+}$and $y^{-}<y<y^{+}$such that $x^{ \pm} \in A, y^{ \pm} \in B$, and $\left|x^{+}-x^{-}\right|<r / R,\left|y^{+}-y^{-}\right|<r / R$. Let us call for a moment $\mathcal{R}$ the rectangle with vertices $\left(x^{ \pm}, y^{ \pm}\right)$. The curve $\omega(\partial \mathcal{R})$ is a small parallelogram around $\omega(x, y)$, with diameter at most $\sqrt{2} r\|M\| / R$; moreover, (4.4) holds for every point of $\partial \mathcal{R}$, thus $\psi=u-\omega$ is uniformly bounded by $2 R \delta r$ on $\partial \mathcal{R}$; therefore, the curve $u(\partial \mathcal{R})$ is entirely contained within distance $2 R \delta r+\sqrt{2} r\|M\| / R$ from $\omega(x, y)$. Since $u$ is a homeomorphism, $u(x, y)$ is inside this curve, hence we get

$$
|\psi(x, y)|=|u(x, y)-\omega(x, y)| \leq 2 R \delta r+\sqrt{2} \frac{r}{R}\|M\|<\varepsilon r,
$$

where the last inequality holds as soon as $R$ has been chosen large enough, depending on $\varepsilon$ and on $\|M\|$, and then $\bar{\delta}$ has been chosen small enough, depending on $\varepsilon$ and $R$, so ultimately on $\varepsilon$ and $\|M\|$. Since $(x, y) \in Q(c, 2 r)$ was an arbitrary point, we obtain (4.1).

To conclude, let us assume that $\operatorname{det} M>0$. Then, observe that the validity of all the inclusions (4.2) holds true as soon as we have the uniform bound

$$
\|u-\omega\|_{L^{\infty}(Q(c, 2 r))}<\nu \varepsilon r,
$$

for a geometrical constant $\nu>0$ only depending on $\|M\|$ and on $\operatorname{det} M$. Notice that this estimate has the same form as (4.1); therefore, to get it we can simply modify the definition of $R$ and of $\bar{\delta}$ done before, in fact one has just to choose $R^{\prime}=R / \nu$ and $\bar{\delta}^{\prime} \leq \nu^{2} \bar{\delta}$. As a consequence, the proof is concluded by replacing the constant $\bar{\delta}(\varepsilon,\|M\|)$ with the smaller constant $\bar{\delta}^{\prime}(\varepsilon,\|M\|$, $\operatorname{det} M)$.

From the above calculations on the Lebesgue squares, we can derive the following useful property about the energy.

Corollary 4.3. Let $Q(c, r)$ be a Lebesgue square corresponding to the matrix $M$ and the constant $\delta$. Then, one has

$$
\mathcal{F}(u, Q) \geq\left(4 \sqrt{\|M\|^{2}+1}-\delta\right) r^{2}, \quad \mathcal{F}\left(u^{-1}, u(Q)\right) \geq|D u|(Q),
$$

and, if $\varepsilon>0$ is given, $\operatorname{det} M \neq 0$, and $\delta \leq \bar{\delta}^{\prime}(\varepsilon,\|M\|$, $\operatorname{det} M)$, it is also

$$
\mathcal{F}\left(u^{-1}, u(Q)\right) \geq|u(Q)|\left(\sqrt{1+\left\|M^{-1}\right\|^{2}}-\alpha\right),
$$

where $\alpha=\alpha(\varepsilon,\|M\|$, det $M)$ is an explicitely computable constant, which converges to 0 if $\varepsilon \searrow 0$ while $\|M\|$ and $1 / \operatorname{det} M$ remain bounded from above. 
Proof. Since $Q=Q(c, r)$ is a Lebesgue square, and since the function $t \mapsto \sqrt{1+t^{2}}$ is 1-Lipschitz, then

$$
\left|\mathcal{F}(u, Q)-\int_{Q} \sqrt{\|M\|^{2}+1}\right| \leq|D u-M|(Q)<\delta r^{2},
$$

from which the first estimate in (4.5) holds. Moreover, by Theorem 3.3 and the fact that $\sqrt{1+t^{2}} \geq t$ we have

$$
\mathcal{F}\left(u^{-1}, u(Q)\right) \geq\left|D u^{-1}\right|(u(Q))=|D u|(Q),
$$

hence also the second estimate in (4.5) is established.

Concerning (4.6), let us fix a constant $\varepsilon>0$, and assume that $\operatorname{det} M \neq 0$ and $\delta \leq$ $\bar{\delta}^{\prime}(\varepsilon,\|M\|$, det $M)$. Let $\omega$ be the affine function given by Lemma 4.2 , and consider the parallelogram $T=\omega(Q(c,(1-\varepsilon) r))$, which is contained in $u(Q)$ by (4.2). Since $D \omega=M$, for every $a, b \in \mathbb{R}^{2}$ one has

$$
|\omega(a)-\omega(b)| \geq \frac{\operatorname{det} M}{\|M\|}|a-b| .
$$

Hence, for any $z \in T$, calling $\tilde{z}=\omega^{-1}(z)$ and $\hat{z}=u^{-1}(z)$, by (4.1) we have

$$
r \varepsilon \geq|\omega(\hat{z})-u(\hat{z})|=|\omega(\hat{z})-\omega(\tilde{z})| \geq \frac{\operatorname{det} M}{\|M\|}|\hat{z}-\tilde{z}|,
$$

which can be rewritten as

$$
\left|u^{-1}(z)-\omega^{-1}(z)\right| \leq \frac{\sqrt{2}}{6} \frac{r \varepsilon\|M\|}{\operatorname{det} M} .
$$

Let then $\mathcal{R} \subseteq T$ be a rectangle, with sides having lengths $\ell_{1}$ and $\ell_{2}$, and directions $\mu, \nu \in \mathbb{S}^{1}$ respectively. If $x$ and $y$ are two points belonging to the two opposite sides of length $\ell_{1}$, with $y-x$ parallel to $\nu,(4.7)$ applied to $x$ and $y$ gives

$$
\left|u^{-1}(x)-u^{-1}(y)\right| \geq\left|\omega^{-1}(x)-\omega^{-1}(y)\right|-2 \frac{r \varepsilon\|M\|}{\operatorname{det} M}=\ell_{2} M^{-1}(\nu)-2 \frac{r \varepsilon\|M\|}{\operatorname{det} M} .
$$

Arguing in the same way for all such couples $x$ and $y$, by integrating we get

$$
\left|D_{\nu} u^{-1}\right|(\mathcal{R}) \geq \ell_{1} \ell_{2} M^{-1}(\nu)-2 \ell_{1} \frac{r \varepsilon\|M\|}{\operatorname{det} M}=|\mathcal{R}|\left(M^{-1}(\nu)-\frac{2 r \varepsilon\|M\|}{\ell_{2} \operatorname{det} M}\right) .
$$

Arguing in the same way for points on the two opposite sides of length $\ell_{2}$, we obtain

$$
\left|D_{\mu} u^{-1}\right|(\mathcal{R}) \geq|\mathcal{R}|\left(M^{-1}(\mu)-\frac{2 r \varepsilon\|M\|}{\ell_{1} \operatorname{det} M}\right) .
$$

By an easy geometrical argument, there are two perpendicular vectors $\mu, \nu \in \mathbb{S}^{1}$ so that, for every $d \leq r \operatorname{det} M /\|M\|$, there are finitely many essentially disjoint rectangles $\mathcal{R}_{j}$, with sides parallel to $\mu$ and $\nu$, all having sides bigger than $d$, so that the union $S:=\cup_{j} \mathcal{R}_{j} \subseteq T$ satisfies

$$
|T \backslash S| \leq 2\|M\| r d .
$$

In particular, we fix $d=r \sqrt{\varepsilon} \operatorname{det} M /\|M\|$, so that also by (4.2)

$$
|S| \geq|T|-2 r^{2} \sqrt{\varepsilon} \operatorname{det} M=\left(4(1-\varepsilon)^{2}-2 \sqrt{\varepsilon}\right) r^{2} \operatorname{det} M \geq \frac{(1-\varepsilon)^{2}-\sqrt{\varepsilon}}{(1+\varepsilon)^{2}}|u(Q)|,
$$

and from the above estimates we obtain

$$
\left|D_{\nu} u^{-1}\right|(S) \geq|S|\left(M^{-1}(\nu)-\frac{2 \sqrt{\varepsilon}\|M\|^{2}}{(\operatorname{det} M)^{2}}\right), \quad\left|D_{\mu} u^{-1}\right|(S) \geq|S|\left(M^{-1}(\mu)-\frac{2 \sqrt{\varepsilon}\|M\|^{2}}{(\operatorname{det} M)^{2}}\right) .
$$


Let us now decompose $D_{\mu} u^{-1}=\nabla_{\mu} u^{-1}+D_{\mu}^{s} u^{-1}$ and $D_{\nu} u^{-1}=\nabla_{\nu} u^{-1}+D_{\nu}^{s} u^{-1}$, with $\nabla_{\mu} u^{-1}$ and $\nabla_{\nu} u^{-1}$ in $L^{1}$ and $D_{\mu}^{s} u^{-1}$ and $D_{\nu}^{s} u^{-1}$ singular measures. Since $u(Q) \supseteq T \supseteq S$, Jensen's inequality, the above estimate and (4.8) give

$$
\begin{aligned}
\mathcal{F}\left(u^{-1}, u(Q)\right) & \geq \mathcal{F}\left(u^{-1}, S\right)=\int_{S} \sqrt{1+\left|\nabla u^{-1}\right|^{2}}+\left|D^{s} u^{-1}\right|(S) \\
& \geq \sqrt{|S|^{2}+\left(\int_{S}\left|\nabla u^{-1}\right|\right)^{2}}+\left|D^{s} u^{-1}\right|(S) \geq \sqrt{|S|^{2}+\left|D u^{-1}\right|(S)^{2}} \\
& \geq \sqrt{|S|^{2}+\left|D_{\nu} u^{-1}\right|(S)^{2}+\left|D_{\mu} u^{-1}\right|(S)^{2}} \geq|S| \sqrt{1+\left\|M^{-1}\right\|^{2}-8 \frac{\sqrt{\varepsilon}\|M\|^{3}}{(\operatorname{det} M)^{3}}} \\
& \geq \frac{(1-\varepsilon)^{2}-\sqrt{\varepsilon}}{(1+\varepsilon)^{2}}|u(Q)| \sqrt{1+\left\|M^{-1}\right\|^{2}-8 \frac{\sqrt{\varepsilon}\|M\|^{3}}{(\operatorname{det} M)^{3}}},
\end{aligned}
$$

from which (4.6) follows. The proof is then concluded.

The following simple result shows that small squares around Lebesgue points for $\nabla u$ are always Lebesgue squares: the same result is well-known for the case of Sobolev mappings, see for instance [13, Lemma 2.2].

Lemma 4.4. Let $x \in \Omega$ be a point of approximate differentiability for $u$, and let $\varepsilon>0$ and $\delta<\bar{\delta}(\varepsilon, \nabla u(x))$ be as in Lemma 4.2. There is then $\bar{r}=\bar{r}(x, \delta)>0$ such that, for every $r<\bar{r}$ and $c \in Q(x, r)$, the square $Q(c, r)$ is a Lebesgue square with matrix $\nabla u(x)$ and constant $\delta$.

Proof. Since $x \in \Omega$ is a point of approximate differentiability for $u$, there exists $\bar{r}=\bar{r}(x, \delta)$ such that for every $r<\bar{r}$ one has $Q(x, 4 r) \subset \subset \Omega$ and

$$
|D u-\nabla u(x)|(Q(x, 4 r))<r^{2} \delta .
$$

Hence, for any $c$ in $Q(x, r)$ we have

$$
|D u-\nabla u(x)|(Q(c, 3 r)) \leq|D u-\nabla u(x)|(Q(x, 4 r))<r^{2} \delta,
$$

thus the thesis is concluded.

Lemma 4.5. Let $\Omega, \Delta \subseteq \mathbb{R}^{2}$ be two bounded open sets, $u \in \mathrm{BV}(\Omega ; \Delta)$ a homeomorphism, and $\varepsilon \ll 1$ fixed. There exists $H=H(u, \varepsilon)$ such that for every $\delta \ll 1 / H$ there exists $\bar{r}=\bar{r}(\delta, H)$ with the property that, for every $r<\bar{r}$, there are essentially disjoint squares $\left\{Q_{i}\right\}_{i=1, \ldots, N}$, all with side $2 r$, each being a Lebesgue square corresponding to a matrix $M_{i}$ and the constant $\delta$, and so that $\left|\Omega \backslash \cup_{i=1}^{N} Q_{i}\right|<\varepsilon$ and for every $1 \leq i \leq N$ one has that either $M_{i}=0$ or $1 / H<\left\|M_{i}\right\|<H$, and either $\operatorname{det} M_{i}=0$ or $1 / H<\operatorname{det} M_{i}<H$.

Proof. For each $H>0$ we define the set

$$
A_{H}:=\{x \in \Omega:\|\nabla u(x)\| \in(0,1 / H) \cup(H,+\infty) \text { or } \operatorname{det}(\nabla u(x)) \in(0,1 / H) \cup(H,+\infty)\} .
$$

Since $A_{H} \subseteq A_{H^{\prime}}$ whenever $H>H^{\prime}$ and $\cap_{H>0} A_{H}=\emptyset$, there is a suitable $H=H(u, \varepsilon) \gg 1$ with $\left|A_{H}\right|<\varepsilon / 3$. Let us now fix any $\delta \ll 1 / H$ : for almost any $x \in \Omega$, we have the constant $\bar{r}(x, \delta)$ from Lemma 4.4; hence, we can find $\bar{r} \ll 1$ such that also the set

$$
B_{H}:=\left\{x \in \Omega \backslash A_{H}: \bar{r}(x, \delta) \leq \bar{r}\right\}
$$

has measure less than $\varepsilon / 3$. Up to decrease the constant $\bar{r}$ if necessary, only depending on $\Omega$, for every $r<\bar{r}$ we can find finitely many essentially disjoint squares $\left\{Q_{j}\right\}_{j=1, \ldots, P}$, all with side $2 r$ 
and centered at points $c_{j} \in \Omega$, such that every square $Q\left(c_{j}, 3 r\right)$ is compactly contained in $\Omega$ and the measure of $\Omega \backslash \cup_{j=1}^{P} Q_{j}$ is less than $\varepsilon / 3$.

Up to renumbering, we have some $N \leq P$ such that the square $Q_{j}$ contains at least a point $x_{j} \in \Omega \backslash\left(A_{H} \cup B_{H}\right)$ if and only if $j \leq N$. By construction, $\Omega \backslash \cup_{j=1}^{N} Q_{j}$ has at most measure $\varepsilon$; moreover, by Lemma 4.4 each $Q_{j}$ with $1 \leq j \leq N$ is a Lebesgue square with constant $\delta$ and matrix $\nabla u\left(x_{j}\right)$, so by the definition of $A_{H}$ the proof is concluded.

\section{Proof of the main Result}

This section is devoted to show our main result, Theorem A. The main step of the construction is the lemma below, showing how to modify a function, slightly in the area strict sense, so to become piecewise affine in a big portion of $\Omega$.

Lemma 5.1. Let $\Omega, \Delta \subseteq \mathbb{R}^{2}$ be two bounded, open sets and $u \in \operatorname{BV}(\Omega ; \Delta)$ be a homeomorphism. For every $\varepsilon>0$ there exists a homeomorphism $v \in \mathrm{BV}(\Omega ; \Delta)$, finitely piecewise affine on a polygon $P \subset \subset \Omega$ with $|\Omega \backslash P|<\varepsilon$, such that $\{v \neq u\} \subset \subset \Omega$, and satisfying

$$
\|v-u\|_{L^{\infty}(\Omega)}+\left\|v^{-1}-u^{-1}\right\|_{L^{\infty}(\Delta)}+\mathcal{F}\left(v^{-1}\right)-\mathcal{F}\left(u^{-1}\right)+|\mathcal{F}(v)-\mathcal{F}(u)|<\varepsilon .
$$

Since the construction is quite involved, we single out the main steps in separate lemmas.

Lemma 5.2. Let $\varepsilon \ll 1$ be given, let $Q=Q(c, r) \subset \subset \Omega$ be a Lebesgue square, corresponding to a matrix $M$ and a constant $\delta \leq \bar{\delta}(\varepsilon,\|M\|)$, with $\delta \leq \bar{\delta}^{\prime}(\varepsilon,\|M\|$, $\operatorname{det} M)$ if $\operatorname{det} M>0$. Then,

$$
|D u|(Q(c, r) \backslash Q(c,(1-\varepsilon) r)) \leq(16\|M\|+10) \varepsilon r^{2}
$$

Proof. By definition of Lebesgue squares, we have

$$
\left|D_{1} u\right|(Q) \leq\left|D_{1} u-M\left(\mathrm{e}_{1}\right)\right|(Q)+4\left|M\left(\mathrm{e}_{1}\right)\right| r^{2} \leq\left(4\left|M\left(\mathrm{e}_{1}\right)\right|+\delta\right) r^{2} .
$$

On the other hand, let us take any $-(1-\varepsilon) r<t<(1-\varepsilon) r$, and call $x_{t}=\left(c_{1}-(1-\varepsilon) r, c_{2}+t\right)$ and $y_{t}=\left(c_{1}+(1-\varepsilon) r, c_{2}+t\right)$ two points on the boundary of $Q(c,(1-\varepsilon) r)$. The $L^{\infty}$ estimate (4.1) ensures that

hence

$$
|| u\left(x_{t}\right)-u\left(y_{t}\right)|-2(1-\varepsilon) r| M\left(\mathrm{e}_{1}\right)|| \leq 2 r \varepsilon,
$$

$$
\left|D_{1} u\right|\left(x_{t} y_{t}\right) \geq\left|u\left(x_{t}\right)-u\left(y_{t}\right)\right| \geq 2(1-\varepsilon) r\left|M\left(\mathrm{e}_{1}\right)\right|-2 r \varepsilon .
$$

Integrating in $t$, we obtain

$$
\left|D_{1} u\right|(Q(c,(1-\varepsilon) r))=\int_{t=-(1-\varepsilon) r}^{(1-\varepsilon) r}\left|D_{1} u\right|\left(x_{t} y_{t}\right) \geq 4(1-\varepsilon)^{2} r^{2}\left|M\left(\mathrm{e}_{1}\right)\right|-4 r^{2} \varepsilon,
$$

which together with (5.3) gives

$$
\left|D_{1} u\right|(Q(c, r) \backslash Q(c,(1-\varepsilon) r)) \leq((8\|M\|+4) \varepsilon+\delta) r^{2} \leq(8\|M\|+5) \varepsilon r^{2} .
$$

Since the very same estimate holds clearly with $D_{2} u$ in place of $D_{1} u$, we have obtained (5.2).

Lemma 5.3. Let $\varepsilon, Q=Q(c, r), M$ and $\delta$ be as in Lemma 5.2, and assume that $\operatorname{det} M \neq 0$. Then there exists a piecewise affine homeomorphism $v: Q \rightarrow \mathbb{R}^{2}$, finitely piecewise affine on a polygon $P \subset \subset Q$ with area at least $(4-8 \varepsilon) r^{2}$, with $v=u$ on $\partial Q$ and

$$
\begin{gathered}
|D u-D v|(Q) \leq 42(1+\|M\|) \varepsilon r^{2}, \\
\mathcal{F}\left(v^{-1}, u(Q)\right) \leq \mathcal{F}\left(u^{-1}, u(Q)\right)+\alpha|u(Q)|+(23\|M\|+31+16 \operatorname{det} M) \varepsilon r^{2},
\end{gathered}
$$

where $\alpha=\alpha(\varepsilon,\|M\|, \operatorname{det} M)$ is the constant given in Corollary 4.3. 
Proof. By construction, we can apply Lemma 4.2, hence we have an affine function $\omega: \mathbb{R}^{2} \times \mathbb{R}^{2}$ with $D \omega=M$ such that (4.1) and (4.2) hold. We can then define $v=\omega$ on the square $P=Q(c,(1-\varepsilon) r)$, so $v$ is in fact affine on the polygon $P$, which has area $4(1-\varepsilon)^{2} r^{2} \geq(4-8 \varepsilon) r^{2}$. Notice that for every $x \in \partial Q(c,(1-\varepsilon) r)$ the point $v(x)$ is in the interior of $u(Q)$ by (4.2). Let us now subdivide $\mathcal{C}=Q(c, r) \backslash Q(c,(1-\varepsilon) r)$ in four rectangles $\mathcal{R}_{j}, 1 \leq j \leq 4$, each with two sides of length $\varepsilon r$ and two sides of length $(2-\varepsilon) r$. We want to define $v$ on $\partial \mathcal{R}_{j}$ for each $j$. Notice that, since $v$ has been already defined on $Q(c,(1-\varepsilon) r)$, and we have to define also $v=u$ on $\partial Q$, it only remains to specify $v$ on four short segments of length $\varepsilon r$. The definition is the following: given any of these short segments, call it $S$, let $S^{\prime} \subseteq S$ be the shortest segment having one endpoint in $\partial Q$, and the other one in $u^{-1}\left(\omega\left(Q\left(c,\left(1-\frac{\varepsilon}{3}\right) r\right)\right)\right)$, which is possible by (4.2). Then, we let $v$ be the function coinciding with $u$ on $S^{\prime}$ and linear on $S \backslash S^{\prime}$. Let us take any rectangle $\mathcal{R}_{j}$, and call $\mathcal{P}$ and $\mathcal{P}^{\prime}$ the internal parts of the Jordan curves $u\left(\partial \mathcal{R}_{j}\right)$ and $v\left(\partial \mathcal{R}_{j}\right)$; let moreover $x$ and $y$ be two points on two opposite sides $\partial \mathcal{R}_{j}$ having one of the two coordinates equal. By construction, thanks to our definition of $v$ on $\partial \mathcal{R}_{j}$ and recalling (4.1), a simple geometric estimate shows that

$$
d_{\mathcal{P}^{\prime}}(x, y) \leq d_{\mathcal{P}}(x, y)+2 \varepsilon r,
$$

so that by integration

$$
\Psi\left(v\left\llcorner\partial \mathcal{R}_{j}\right) \leq \Psi\left(u\left\llcorner\partial \mathcal{R}_{j}\right)+4 \varepsilon r^{2} .\right.\right.
$$

Hence, by Proposition 3.8 we can extend $v$ to the interior of $\mathcal{R}_{j}$ as a homeomorphism, satisfying

$$
\int_{\mathcal{R}_{j}}|D v| \leq \Psi\left(v\left\llcorner\partial \mathcal{R}_{j}\right) \leq \Psi\left(u\left\llcorner\partial \mathcal{R}_{j}\right)+4 \varepsilon r^{2} \leq \sqrt{2}|D u|\left(\mathcal{R}_{j}\right)+4 \varepsilon r^{2},\right.\right.
$$

where we have also taken Lemma 3.5 in account. Adding this inequality for the four rectangles and keeping in mind Lemma 5.2 we obtain

$$
\int_{\mathcal{C}}|D v| \leq \sqrt{2}|D u|(\mathcal{C})+16 \varepsilon r^{2} \leq(23\|M\|+31) \varepsilon r^{2} .
$$

In the smaller square $P$, instead, we have

$$
|D u-D v|(P)=|D u-M|(P) \leq \delta r^{2}<\varepsilon r^{2} .
$$

Putting the last two estimates together we get

$$
|D u-D v|(Q) \leq \varepsilon r^{2}+|D u|(\mathcal{C})+|D v|(\mathcal{C}) \leq(39\|M\|+42) \varepsilon r^{2},
$$

which is stronger than (5.4). We have then now to prove (5.5).

First of all, since $v=\omega$ on $P$, we have

$$
\mathcal{F}\left(v^{-1}, v(P)\right)=\sqrt{1+\left\|M^{-1}\right\|^{2}}|v(P)| .
$$

Moreover, by (4.2) we have

$$
v(P)=\omega(Q(c,(1-\varepsilon) r)), \quad v(Q)=u(Q) \subseteq \omega(Q(c,(1+\varepsilon) r)),
$$

so that

$$
|v(Q) \backslash v(P)|=|v(Q)|-|v(P)| \leq 16 \varepsilon r^{2} \operatorname{det} M .
$$

As a consequence, since $\sqrt{1+t^{2}} \leq 1+t$ and by Theorem 3.3 and (5.6) we have

$$
\begin{aligned}
\mathcal{F}\left(v^{-1}, v(Q) \backslash v(P)\right) & \leq\left|D v^{-1}\right|(v(Q) \backslash v(P))+|v(Q) \backslash v(P)| \leq|D v|(\mathcal{C})+16 \varepsilon r^{2} \operatorname{det} M \\
& \leq(23\|M\|+31+16 \operatorname{det} M) \varepsilon r^{2} .
\end{aligned}
$$

Putting this estimate together with (5.7) and with (4.6) from Corollary 4.3, we get (5.5). 
Lemma 5.4. Let $\varepsilon, Q=Q(c, r), M$ and $\delta$ be as in Lemma 5.2, and assume that $M=0$. Then there exists a piecewise affine homeomorphism $v: Q \rightarrow \mathbb{R}^{2}$, finitely piecewise affine on a polygon $P \subset \subset Q$ with area at least $(4-8 \varepsilon) r^{2}$, with $v=u$ on $\partial Q$ and

$$
\begin{gathered}
|D u-D v|(Q) \leq 3 \varepsilon r^{2}, \\
\mathcal{F}\left(v^{-1}, u(Q)\right) \leq \mathcal{F}\left(u^{-1}, u(Q)\right)+2 \varepsilon r^{2} .
\end{gathered}
$$

Proof. Let $0<t<\varepsilon$ be a number, to be specified in a moment. Similarly to the previous lemma, we call $P=Q(c,(1-t) r)$ a square slightly smaller than $Q$, which has of course area larger than $(4-8 \varepsilon) r^{2}$, and we subdivide $\mathcal{C}=Q \backslash P$ in four rectangles $\mathcal{R}_{j}, 1 \leq j \leq 4$. Let us now call $\Gamma$ the union of the boundaries of the square $P$ and of the rectangles $\mathcal{R}_{j}$, and $\Gamma_{0}$ the boundary of $P$. We can assume that the restriction of $u$ to $\Gamma \backslash \partial Q$ belongs to $W^{1,1}$, since this is true for every choice of $t$ outside a negligible subset of the interval $(0, \varepsilon)$. As a consequence, for every $\eta>0$ there exists $\xi<t r$ such that every segment $x y$ contained in $\Gamma \backslash \partial Q$ with length smaller than $\xi$ has image, under $u$, with length less than $\eta$.

Let then $\eta \ll 1$ be a constant, to be chosen in a moment, and let $\xi>0$ be the corresponding constant. Applying Lemma 3.1 with our choice of $\Gamma, \Gamma_{0}$ and $\xi$, and being $g$ the restriction of $u$ to $\Gamma$, we obtain an injective function $\varphi: \Gamma \rightarrow \mathbb{R}^{2}$. Since $\xi<t r$, we have that $\varphi=u$ on $\partial Q$, and on the other hand $\varphi$ is piecewise linear on $\partial P$. By construction, $\varphi$ is a $\eta$-linearization of $g$ on each of the four rectangles $\partial \mathcal{R}_{j}$ as well as on $\partial P$, according to Definition 3.6. As a consequence, by Lemma 3.7 we have

$$
\Psi\left(\varphi\left\llcorner\partial \mathcal{R}_{j}\right) \leq \Psi\left(u\left\llcorner\partial \mathcal{R}_{j}\right)+4 \eta r \quad \forall 1 \leq j \leq 4, \quad \Psi(\varphi\llcorner\partial P) \leq \Psi(u\llcorner\partial P)+8 \eta r .\right.\right.
$$

Thanks to Proposition 3.8, we can find a piecewise affine extension $v$ of $\varphi$ on each $\mathcal{R}_{j}$ and on $P$ in such a way that, also by Lemma 3.5 ,

$$
\begin{aligned}
\int_{Q}|D v| & =\int_{P}|D v|+\sum_{j=1}^{4} \int_{\mathcal{R}_{j}}|D v| \leq \Psi\left(\varphi\llcorner\partial P)+\sum_{j=1}^{4} \Psi\left(\varphi\left\llcorner\partial \mathcal{R}_{j}\right)\right.\right. \\
& \leq \Psi\left(u\llcorner\partial P)+\sum_{j=1}^{4} \Psi\left(u\left\llcorner\partial \mathcal{R}_{j}\right)+24 \eta r \leq \sqrt{2}|D u|(Q)+24 \eta r .\right.\right.
\end{aligned}
$$

Notice that $v=u$ on $\partial Q$, and that $v$ is finitely piecewise affine on $P$ since $\varphi$ is piecewise linear on $\partial P$. Then we have only to take care of (5.8) and (5.9). Since $Q$ is a Lebesgue square corresponding to $M=0$, by definition we have $|D u|(Q)<\delta r^{2}<\varepsilon r^{2}$, hence

$$
|D u-D v|(Q) \leq|D u|(Q)+|D v|(Q) \leq(1+\sqrt{2})|D u|(Q)+24 \eta r \leq 3 \varepsilon r^{2},
$$

where the last inequality is true up to have chosen $\eta$ small enough. We have then proved (5.8).

Recall now that, by (4.1) of Lemma 4.2 and since $M=0$, the set $u(Q)$ has area less than $\pi \varepsilon^{2} r^{2}$. As a consequence, since $\sqrt{1+t^{2}} \leq 1+t$ and by Theorem 3.3 we have

$$
\begin{aligned}
\mathcal{F}\left(v^{-1}, u(Q)\right)-\mathcal{F}\left(u^{-1}, u(Q)\right) & \leq \mathcal{F}\left(v^{-1}, u(Q)\right) \leq\left|D v^{-1}\right|(v(Q))+|u(Q)| \\
& \leq|D v|(Q)+\pi \varepsilon^{2} r^{2} \leq \sqrt{2} \varepsilon r^{2}+24 \eta r+\pi \varepsilon^{2} r^{2},
\end{aligned}
$$

from which also (5.9) follows if $\eta$ is chosen small enough. The proof is then complete.

Lemma 5.5. Let $\varepsilon, Q=Q(c, r), M$ and $\delta$ be as in Lemma 5.2, and assume that $\operatorname{det} M=0$ but $M \neq 0$. Calling $\bar{\varepsilon}$ and $K$ the constants of Theorem 3.2, assume in addition that $\delta<\|M\| \varepsilon \bar{\varepsilon}$. 
Then there exists a piecewise affine homeomorphism $v: Q \rightarrow \mathbb{R}^{2}$, finitely piecewise affine on a polygon $P \subset \subset Q$ with area at least $(4-8 \varepsilon) r^{2}$, with $v=u$ on $\partial Q$ and

$$
\begin{gathered}
|D u-D v|(Q) \leq 39\left(1+K \frac{\delta}{\varepsilon^{2}}\right)(1+\|M\|) \varepsilon r^{2}, \\
\mathcal{F}\left(v^{-1}, u(Q)\right) \leq \mathcal{F}\left(u^{-1}, u(Q)\right)+43\left(1+K \frac{\delta}{\varepsilon^{2}}\right)(1+\|M\|) \varepsilon r^{2} .
\end{gathered}
$$

Proof. Let $0<t<\varepsilon$ be a number, to be fixed in a moment. We will call again $P=Q(c,(1-t) r)$ the slightly smaller square, and we will again subdivide $\mathcal{C}=Q \backslash P$ in four rectangles $\mathcal{R}_{j}$, $1 \leq j \leq 4$. Moreover, we call again $\Gamma_{0}$ the boundary of $P$ and $\Gamma$ the union of the boundaries of $P$ and of the rectangles $\mathcal{R}_{j}$. As already noticed, the restriction of $u$ to $\Gamma \backslash \partial Q$ belongs to $W^{1,1}$ for all $t$ except those contained in a negligible subset of $(0, \varepsilon)$. Moreover, if we call $S_{t}$ the boundary of $P$, by Fubini Theorem we have

$$
\delta r^{2}>|D u-M|(\mathcal{C})=r \int_{t=0}^{\varepsilon}|D u-M|\left(S_{t}\right) d t,
$$

hence we can select some $0<t<\varepsilon$ such that not only $u$ belongs to $W^{1,1}(\Gamma \backslash \partial Q)$, but also

$$
|D u-M|(\partial P) \leq \frac{\delta}{\varepsilon} r .
$$

Let again $\eta \ll 1$ be a small constant, to be chosen in a moment, and let $\xi<t r$ such that every segment $x y$ contained in $\Gamma \backslash \partial Q$ with length smaller than $\xi$ has image, under $u$, with length less than $\eta$. Applying Lemma 3.1, with $g$ being the restriction of $u$ to $\Gamma$, we obtain an injective function $\varphi: \Gamma \rightarrow \mathbb{R}^{2}$, and by construction $\varphi=u$ on $\partial Q$ and $\varphi$ is a $\eta$-linearization of $g$ on the boundary of every $\mathcal{R}_{j}$. As in the previous lemma, then, we have

$$
\Psi\left(\varphi\left\llcorner\partial \mathcal{R}_{j}\right) \leq \Psi\left(u\left\llcorner\partial \mathcal{R}_{j}\right)+4 \eta r \quad \forall 1 \leq j \leq 4,\right.\right.
$$

hence Proposition 3.8 provides us with a piecewise affine extension $v$ of $\varphi$ inside each $\mathcal{R}_{j}$ which, by Lemma 3.5, satisfies

$$
|D v|(\mathcal{C}) \leq \sum_{j=1}^{4} \Psi\left(\varphi\left\llcorner\partial \mathcal{R}_{j}\right) \leq \sum_{j=1}^{4} \Psi\left(u\left\llcorner\partial \mathcal{R}_{j}\right)+16 \eta r \leq \sqrt{2}|D u|(\mathcal{C})+16 \eta r .\right.\right.
$$

Concerning the square $P$ we need to argue in a different way: more precisely, $\varphi$ is piecewise linear on $\partial P$, and since $\varphi$ is a linearization of $g$, then by (5.12)

$$
|D \varphi-M \cdot \tau|(\partial P) \leq|D g-M \cdot \tau|(\partial P) \leq|D u-M|(\partial P) \leq \frac{\delta}{\varepsilon} r .
$$

Notice that the first inequality is true because, for each segment $x y \subseteq \partial P$ for which $\varphi$ is linear on $x y$ and coincides with $g$ on $x$ and on $y$, the vector $D \varphi-M$ is constant and coincides with the average of the vector $D g-M$, thus by concavity of the distance we clearly have $|D \varphi-M \cdot \tau|(x y) \leq|D g-M \cdot \tau|(x y)$.

Since $\delta r / \varepsilon<\|M\| \bar{\varepsilon} r$, by (5.14) we can apply Theorem 3.2, which provides us with a finitely piecewise extension $v$ of $\varphi$ on $P$ satisfying

$$
|D v-M|(P) \leq K \frac{\delta}{\varepsilon} r^{2} .
$$

The function $v$ on the whole $Q$ is then a BV extension of $u$ on $\partial Q$, which is finitely piecewise affine on the polygon $P$, having area at least $(4-8 \varepsilon) r^{2}$. Moreover, putting together the last 
estimate and (5.13), also by Lemma 5.2 and the definition of Lebesgue squares we obtain

$$
\begin{aligned}
|D v-D u|(Q) & \leq|D v|(\mathcal{C})+|D u|(\mathcal{C})+|D v-M|(P)+|D u-M|(P) \\
& \leq(39\|M\|+25) \varepsilon r^{2}+16 \eta r+K \frac{\delta}{\varepsilon} r^{2}+\varepsilon r^{2},
\end{aligned}
$$

which is stronger than (5.10) up to have chosen $\eta$ small enough, depending on $\varepsilon$.

To conclude, recall that by Lemma 4.2 there exists an affine function $\omega$ with $D \omega=M$ such that $\|u-\omega\|_{L^{\infty}(Q)} \leq \varepsilon r$. Since $\omega(Q)$ is a segment with length $\|M\| r$, we deduce that $|u(Q)| \leq 4(\|M\|+1) \varepsilon r^{2}$, thus

$$
\begin{aligned}
\mathcal{F}\left(v^{-1}, u(Q)\right)-\mathcal{F}\left(u^{-1}, u(Q)\right) & \leq\left|D v^{-1}\right|(u(Q))+|u(Q)|-\left|D u^{-1}\right|(u(Q)) \\
& \leq|D v|(Q)-|D u|(Q)+4(\|M\|+1) \varepsilon r^{2}
\end{aligned}
$$

hence (5.11) follows from (5.10) and the proof is concluded.

We are now ready to prove Lemma 5.1.

Proof (of Lemma 5.1). First of all, we apply Lemma 4.5 with the constant $\varepsilon_{1}=\varepsilon /(1+2|\Omega|)$, thus finding the constant $H \gg 1$. Then, we define $\varepsilon_{2}=\varepsilon_{2}(u, \varepsilon, H) \ll \varepsilon_{1}$ with the property that

$$
\alpha\left(\varepsilon_{2},\|M\|, \operatorname{det} M\right)<\frac{\varepsilon}{8|\Delta|} \quad \forall M \in \mathbb{R}^{2 \times 2}:(\|M\|, \operatorname{det} M) \in(1 / H, H)^{2},
$$

and that

$$
22(1+2 H) \varepsilon_{2}|\Omega|<\frac{\varepsilon}{8} .
$$

Moreover, calling $\bar{\varepsilon}$ and $K$ the constants of Theorem 3.2, let $\delta=\delta\left(\varepsilon_{2}, H\right) \ll 1 / H$ be such that

$$
\begin{array}{cl}
\delta<\bar{\delta}\left(\varepsilon_{2},\|M\|\right) & \forall M \in \mathbb{R}^{2 \times 2}:\|M\| \in\{0\} \cup(1 / H, H), \\
\delta<\bar{\delta}^{\prime}\left(\varepsilon_{2},\|M\|, \operatorname{det} M\right) & \forall M \in \mathbb{R}^{2 \times 2}:(\|M\|, \operatorname{det} M) \in(1 / H, H)^{2},
\end{array}
$$

and

$$
\delta<\min \left\{\frac{\varepsilon_{2} \bar{\varepsilon}}{H}, \frac{\varepsilon_{2}^{2}}{K}\right\} .
$$

We use now our choice of $\delta$ to apply Lemma 4.5, finding the constant $\bar{r}=\bar{r}(\delta, H)=\bar{r}(u, \varepsilon, H)$. Then, we pick some $r<\bar{r}$ such that

$$
|u(x)-u(y)|<\frac{\varepsilon}{4} \quad \forall x, y \in \Omega:|y-x|<2 \sqrt{2} r, \quad r<\frac{\varepsilon}{16} \sqrt{2},
$$

and we finally use this $r$ in Lemma 4.5 to get the squares $\left\{Q_{i}\right\}_{i=1, \ldots, N}$ and the corresponding matrices $M_{i}$; keep in mind that, by construction,

$$
\left|\Omega \backslash \cup_{i=1}^{N} Q_{i}\right|<\varepsilon_{1}=\frac{\varepsilon}{1+2|\Omega|} .
$$

By (5.17) and (5.18) to every square $Q_{i}$, with constants $\varepsilon_{2}$ and $\delta$ and matrix $M_{i}$, we can apply either Lemma 5.3, or Lemma 5.4, or Lemma 5.5, respectively if $\operatorname{det} M_{i} \neq 0$, or $M_{i}=0$, or $\operatorname{det} M_{i}=0 \neq\left\|M_{i}\right\|$, thus finding the functions $v_{i}: Q_{i} \rightarrow \mathbb{R}^{2}$. We let $v \in \operatorname{BV}(\Omega ; \Delta)$ be the function coinciding with $v_{i}$ on every $Q_{i}$, while $v=u$ outside of the union of the squares.

It is true by construction that $v$ is a homeomorphism and that $\{v \neq u\} \subseteq \cup_{i=1}^{N} Q_{i} \subset \subset \Omega$. Moreover, every square $Q_{i}$ contains a polygon $P_{i}$ with $\left|Q_{i} \backslash P_{i}\right|<2 \varepsilon_{2}\left|Q_{i}\right|$ on which $v_{i}$ is finitely piecewise affine, hence $v$ is finitely piecewise affine on the polygon $P=\cup_{i=1}^{N} P_{i}$, and by $(5.20)$

$$
|\Omega \backslash P|=\left|\Omega \backslash \cup_{i=1}^{N} Q_{i}\right|+\sum_{i=1}^{N}\left|Q_{i} \backslash P_{i}\right|<\frac{\varepsilon}{1+2|\Omega|}+2 \varepsilon_{2}\left|\cup_{i=1}^{N} Q_{i}\right|<\varepsilon .
$$


To conclude, we have then only to show (5.1).

First of all, notice that for every square $Q_{i}$ we have $v=u$ on $\partial Q_{i}$, hence for every $x \in Q_{i}$ it must be $|v(x)-u(x)|<\operatorname{diam}\left(u\left(Q_{i}\right)\right)<\varepsilon / 4$ by the first property in (5.19), so we deduce

$$
\|v-u\|_{L^{\infty}(\Omega)}<\frac{\varepsilon}{4} .
$$

Analogously, for every $1 \leq i \leq N$ and for every $z \in u\left(Q_{i}\right)$, we have that both $u^{-1}(z)$ and $v^{-1}(z)$ belong to $Q_{i}$, hence their distance is at most $2 \sqrt{2} r$, so the second property in (5.19) gives

$$
\left\|v^{-1}-u^{-1}\right\|_{L^{\infty}(\Delta)}<\frac{\varepsilon}{4}
$$

Concerning $\mathcal{F}\left(v^{-1}\right)-\mathcal{F}\left(u^{-1}\right)$, up to renumbering we can assume that $\operatorname{det} M_{i} \neq 0$ if and only if $i \leq N_{1}$ for some $N_{1} \leq N$. Then, in every square $Q_{i}$ we can use either (5.5), or (5.9), or (5.11), which also keeping in mind (5.15), (5.16) and (5.18) gives

$$
\begin{aligned}
\mathcal{F}\left(v^{-1}\right)- & \mathcal{F}\left(u^{-1}\right)=\sum_{i=1}^{N} \mathcal{F}\left(v_{i}^{-1}, u\left(Q_{i}\right)\right)-\mathcal{F}\left(u^{-1}, u\left(Q_{i}\right)\right) \\
& \leq \sum_{i=1}^{N_{1}} \alpha\left(\varepsilon_{2},\left\|M_{i}\right\|, \operatorname{det} M_{i}\right)\left|u\left(Q_{i}\right)\right|+\sum_{i=1}^{N} 43\left(1+\left\|M_{i}\right\|+\operatorname{det} M_{i}\right)\left(1+\frac{K \delta}{\varepsilon_{2}^{2}}\right) \varepsilon_{2} r^{2} \\
& \leq \frac{\varepsilon}{8}+11(1+2 H)\left(1+\frac{K \delta}{\varepsilon_{2}^{2}}\right) \varepsilon_{2}|\Omega|<\frac{\varepsilon}{4} .
\end{aligned}
$$

Finally, concerning $\mathcal{F}(v)-\mathcal{F}(u)$, since $t \mapsto \sqrt{1+t^{2}}$ is 1 -Lipschitz we can use (5.4), (5.8) and (5.10), together with (5.16) and (5.18), to get

$$
|\mathcal{F}(v)-\mathcal{F}(u)|=\sum_{i=1}^{N}\left|\mathcal{F}\left(v_{i}, Q_{i}\right)-\mathcal{F}\left(u, Q_{i}\right)\right| \leq \sum_{i=1}^{N}\left|D v_{i}-D u\right|\left(Q_{i}\right)<\frac{\varepsilon}{4} .
$$

This inequality, together with (5.21), (5.22) and (5.23), gives (5.1), thus concluding the proof.

We can now conclude the paper by showing our main result, which can be obtained applying twice Lemma 5.1 if $\Omega$ is bounded, while the general case needs an approximation argument.

Proof (of Theorem A). We divide the proof in two steps.

Step I. The case when $\Omega$ and $\Delta$ are bounded.

Let us first assume that $\Omega$ and $\Delta$ are bounded. In this case, let $j \in \mathbb{N}$ be fixed, and let $\varepsilon=1 / j$. We apply Lemma 5.1 to $u$, finding a polygon $P_{1} \subset \subset \Omega$ and a homeomorphism $\varphi_{1}: \Omega \rightarrow \mathbb{R}^{2}$, finitely piecewise affine on $P_{1}$, such that $\left\{\varphi_{1} \neq u\right\} \subset \subset \Omega,\left|\Omega \backslash P_{1}\right|<\varepsilon$, and (5.1) holds. Let us now apply again Lemma 5.1 to the restriction of $\varphi_{1}^{-1}$ to $\Delta \backslash \varphi_{1}\left(P_{1}\right)$, finding another BV homeomorphism $\varphi_{2}: \Delta \backslash \varphi_{1}\left(P_{1}\right) \rightarrow \mathbb{R}^{2}$, finitely piecewise affine on some polygon $P_{2} \subset \subset \Delta \backslash \varphi_{1}\left(P_{1}\right)$ with $\Delta \backslash\left(\varphi_{1}\left(P_{1}\right) \cup P_{2}\right)<\varepsilon$ and $\left\{\varphi_{2} \neq \varphi_{1}^{-1}\right\} \subset \subset \Delta \backslash \varphi_{1}\left(P_{1}\right)$, and again such that (5.1) holds. Let us now define $\varphi_{3}: \Omega \rightarrow \Delta$ the function which coincides with $\varphi_{2}^{-1}$ on $\Omega \backslash P_{1}$ and with $\varphi_{1}$ on $P_{1}$; by construction, it is a homeomorphism which satisfies

$$
\left\|\varphi_{3}-u\right\|_{L^{\infty}(\Omega)}+\left\|\varphi_{3}^{-1}-u^{-1}\right\|_{L^{\infty}(\Delta)}+\mathcal{F}\left(\varphi_{3}^{-1}\right)-\mathcal{F}\left(u^{-1}\right)+\mathcal{F}\left(\varphi_{3}\right)-\mathcal{F}(u)<2 \varepsilon,
$$

and which is finitely piecewise affine on the polygon $P=P_{1} \cup \varphi_{2}^{-1}\left(P_{2}\right)$. By construction,

$$
|\Omega \backslash P|<\varepsilon, \quad\left|\Delta \backslash \varphi_{3}(P)\right|<\varepsilon .
$$

Keep in mind that $P \subset \subset \Omega$, and that $\varphi_{3}$ is piecewise linear on $\partial P$. Then, calling $\Omega^{-}=\Omega \backslash P$ and $\Delta^{-}=\Delta \backslash \varphi_{3}(P)$ for brevity, we apply Theorem 3.9 to the restriction of $\varphi_{3}$ to $\Omega^{-}$, finding a 
countably piecewise affine homeomorphism $\varphi_{4}: \Omega^{-} \rightarrow \Delta^{-}$which coincides with $\varphi_{3}$ on $\partial P$ and which is finitely piecewise affine on a neighborhood of $\partial P$, and such that

$$
\begin{array}{cc}
\left\|\varphi_{4}-\varphi_{3}\right\|_{L^{\infty}\left(\Omega^{-}\right)}<\varepsilon, & \left\|\varphi_{4}^{-1}-\varphi_{3}^{-1}\right\|_{L^{\infty}\left(\Delta^{-}\right)}<\varepsilon, \\
|| D \varphi_{4}\left|\left(\Omega^{-}\right)-\right| D \varphi_{3}\left|\left(\Omega^{-}\right)\right|<\varepsilon, & || D \varphi_{4}^{-1}\left|\left(\Delta^{-}\right)-\right| D \varphi_{3}^{-1}\left|\left(\Delta^{-}\right)\right|<\varepsilon .
\end{array}
$$

Finally, define $v_{j}: \Omega \rightarrow \Delta$ the function which coincides with $\varphi_{3}$ on $P$ and with $\varphi_{4}$ on $\Omega^{-}$. By construction, this is a countably piecewise affine BV homeomorphism, uniformly coinciding with $u$ on $\partial \Omega$ and finitely piecewise affine where possible. Notice that

$$
\left\|v_{j}-u\right\|_{L^{\infty}(\Omega)}<3 \varepsilon, \quad\left\|v_{j}^{-1}-u^{-1}\right\|_{L^{\infty}(\Delta)}<3 \varepsilon,
$$

and moreover by (5.25) and (5.26), also recalling (2.1), we have

$$
\mathcal{F}\left(v_{j}\right)=\mathcal{F}\left(\varphi_{3}, P\right)+\mathcal{F}\left(\varphi_{4}, \Omega^{-}\right)=\mathcal{F}\left(\varphi_{3}, \Omega\right)+\mathcal{F}\left(\varphi_{4}, \Omega^{-}\right)-\mathcal{F}\left(\varphi_{3}, \Omega^{-}\right) \leq \mathcal{F}\left(\varphi_{3}, \Omega\right)+2 \varepsilon .
$$

In the very same way,

$$
\mathcal{F}\left(v_{j}^{-1}\right) \leq \mathcal{F}\left(\varphi_{3}^{-1}, \Delta\right)+2 \varepsilon
$$

hence by (5.24) we deduce

$$
\mathcal{F}\left(v_{j}\right)+\mathcal{F}\left(v_{j}^{-1}\right) \leq \mathcal{F}(u)+\mathcal{F}\left(u^{-1}\right)+\frac{6}{j} .
$$

In particular, $\left\{v_{j}\right\}$ and $\left\{v_{j}^{-1}\right\}$ are bounded in BV, thus by (5.27) they converge weakly* to $u$ and $u^{-1}$ respectively. Since, as noticed with Lemma 2.3 , the functional $\mathcal{F}$ is lower semicontinuous with respect to the weak* convergence, $(5.28)$ implies that $\mathcal{F}\left(v_{j}\right)$ and $\mathcal{F}\left(v_{j}^{-1}\right)$ converge to $\mathcal{F}(u)$ and $\mathcal{F}\left(u^{-1}\right)$ respectively, hence they converge in the area strict sense since $\Omega$ and $\Delta$ are bounded. The proof is then concluded in this case.

\section{Step II. The general case.}

Let us now give the proof in the general case, when $\Omega$ and $\Delta$ might be unbounded. Let $j \in \mathbb{N}$ be fixed, and let $\Omega_{j} \subset \subset \Omega$ be an open, smooth set such that

$$
|D u|\left(\Omega \backslash \Omega_{j}\right)<\varepsilon,
$$

where again we write for brevity $\varepsilon=1 / j$. Calling $\Delta_{j}=u\left(\Omega_{j}\right)$, we can applying the result of Step I to the function $u$ on the set $\Omega_{j}$, finding a sequence of functions $\left\{\varphi_{j, n}\right\}_{n \in \mathbb{N}} \subseteq \operatorname{BV}\left(\Omega_{j}, \Delta_{j}\right)$, which converge uniformly and in the area strict sense to $u$ and whose inverses converge uniformly and in the area strict sense to $u^{-1}$. By Lemma 2.3, we can find $n=n(j)$ such that, writing $\varphi_{j}=\varphi_{j, n(j)}$, one has

$$
\left\|\varphi_{j}-u\right\|_{L^{1}\left(\Omega_{j}\right)}+\left\|\varphi_{j}-u\right\|_{L^{\infty}\left(\Omega_{j}\right)}+\left\|\varphi_{j}^{-1}-u^{-1}\right\|_{L^{1}\left(\Delta_{j}\right)}+\left\|\varphi_{j}^{-1}-u^{-1}\right\|_{L^{\infty}\left(\Delta_{j}\right)}<\varepsilon,
$$

and moreover one can write $D \varphi_{j}=\mu^{\prime}+\nu^{\prime}$ so that

$$
\left|\mu^{\prime}-\nabla u\right|\left(\Omega_{j}\right)<\varepsilon, \quad|| \nu^{\prime}\left|\left(\Omega_{j}\right)-\right| D^{s} u\left|\left(\Omega_{j}\right)\right|<\varepsilon .
$$

Let now $P \subset \subset \Omega_{j}$ be a polygon such that

$$
|D u|\left(\Omega_{j} \backslash P\right)+\left|D \varphi_{j}\right|\left(\Omega_{j} \backslash P\right)<\varepsilon,
$$

and call $\Omega^{-}=\Omega \backslash P$ and $\Delta^{-}=\Delta \backslash \varphi_{j}(P)$. Let then $v: \Omega^{-} \rightarrow \Delta^{-}$be the function which coincides with $\varphi_{j}$ on $\Omega_{j} \backslash P$, and with $u$ on $\Omega \backslash \Omega_{j}$. By construction, (5.29) and (5.32), this is a BV homeomorphism with

$$
|D v|\left(\Omega^{-}\right)<2 \varepsilon
$$


and it is continuous up to $\partial P$, being piecewise linear there. We can then apply Theorem 3.9 to $v$, finding a $\mathrm{BV}$ homeomorphism $\tilde{v}: \Omega^{-} \rightarrow \Delta^{-}$such that

$$
\|\tilde{v}-v\|_{L^{1}\left(\Omega^{-}\right)}+\|\tilde{v}-v\|_{L^{\infty}\left(\Omega^{-}\right)}+\left\|\tilde{v}^{-1}-v^{-1}\right\|_{L^{1}\left(\Delta^{-}\right)}+\left\|\tilde{v}^{-1}-v^{-1}\right\|_{L^{\infty}\left(\Delta^{-}\right)}<\varepsilon,
$$

and with

$$
|D \tilde{v}|\left(\Omega^{-}\right) \leq|D v|\left(\Omega^{-}\right)+\varepsilon<3 \varepsilon,
$$

where we have also used (5.33). Let us finally define $v_{j}: \Omega \rightarrow \Delta$ the function which coincides with $\varphi_{j}$ on $P$, and with $\tilde{v}$ on $\Omega^{-}$. By construction, this is a piecewise affine BV homeomorphism, uniformly coinciding with $u$ on $\partial \Omega$ and finitely piecewise affine where possible. By (5.30) and (5.34), we have that $v_{j}$ and $v_{j}^{-1}$ converge uniformly and in $L^{1}$ to $u$ and $u^{-1}$ respectively. Since $\left|D v_{j}\right|\left(\Omega_{j}\right)$ and $\left|D v_{j}\right|\left(\Omega^{-}\right)$are bounded by (5.31) and (5.35), then also by Theorem $3.3 v_{j}$ and $v_{j}^{-1}$ are bounded in BV, so they weak* converge to $u$ and $u^{-1}$. Finally, let us call $\nu_{j}=\nu^{\prime}\llcorner P$, and $\mu_{j}=D v_{j}-\nu_{j}$, so we have decomposed $D v_{j}=\mu_{j}+\nu_{j}$. We have

$$
\left|\nu_{j}-\nu^{\prime}\right|(\Omega)=\left|\nu^{\prime}\right|\left(\Omega_{j} \backslash P\right) \leq\left|D \varphi_{j}\right|\left(\Omega_{j} \backslash P\right)<\varepsilon
$$

by (5.32), hence by (5.29) and (5.31)

$$
|| \nu_{j}|(\Omega)-| D^{s} u|(\Omega)| \leq|| \nu^{\prime}|(\Omega)-| D^{s} u|(\Omega)|+\varepsilon \leq|| \nu^{\prime}\left|\left(\Omega_{j}\right)-\right| D^{s} u\left|\left(\Omega_{j}\right)\right|+2 \varepsilon<3 \varepsilon .
$$

Moreover, we have

$$
\begin{aligned}
\mu_{j}-\nabla u & =D v_{j}-\nu_{j}-\nabla u=D \varphi_{j}\left\llcorner P+D \tilde{v}-\nu^{\prime}-\left(\nu_{j}-\nu^{\prime}\right)-\nabla u\left\llcorner\Omega_{j}-\nabla u\left\llcorner\left(\Omega \backslash \Omega_{j}\right)\right.\right.\right. \\
& =D \varphi_{j}-D \varphi_{j}\left\llcorner\left(\Omega_{j} \backslash P\right)+D \tilde{v}-\nu^{\prime}-\left(\nu_{j}-\nu^{\prime}\right)-\nabla u\left\llcorner\Omega_{j}-\nabla u\left\llcorner\left(\Omega \backslash \Omega_{j}\right)\right.\right.\right. \\
& =\mu^{\prime}-\nabla u\left\llcorner\Omega_{j}-D \varphi_{j}\left\llcorner\left(\Omega_{j} \backslash P\right)+D \tilde{v}-\left(\nu_{j}-\nu^{\prime}\right)-\nabla u\left\llcorner\left(\Omega \backslash \Omega_{j}\right),\right.\right.\right.
\end{aligned}
$$

so by $(5.31),(5.32),(5.35),(5.36)$ and $(5.29)$ we get

$$
\left|\mu_{j}-\nabla u\right|(\Omega)<7 \varepsilon \text {. }
$$

This equation, together with (5.37), ensures that $v_{j} \stackrel{\text { area }}{\longrightarrow} u$, hence the proof is concluded.

\section{REFERENCES}

[1] L. Ambrosio, N. Fusco, D. Pallara, Functions of Bounded Variation and Free Discontinuity Problems, Oxford University Press (2000).

[2] H. Brezis, Convergence in $\mathcal{D}^{1}$ and in $L^{1}$ under strict convexity, in Boundary value problems for partial differential equations and applications, dedicated to E. Magenes, (C. Baiocchi and J.L. Lions eds.), Masson, 1993, 43-52.

[3] D. Campbell, Diffeomorphic approximation of Planar Sobolev Homeomorphisms in Orlicz-Sobolev spaces, preprint (2016).

[4] L. D'Onofrio \& R. Schiattarella, On the total variations for the inverse of a BV-homeomorphism, Adv. Calc. Var. 6 (2013), 321-328.

[5] S. Daneri \& A. Pratelli, Smooth approximation of bi-Lipschitz orientation-preserving homeomorphisms, Ann. Inst. H. Poincaré Anal. Non Linéaire, 31, n. 3 (2014), 567-589.

[6] S. Delladio, Lower semicontinuity and continuity of functions of measures with respect to the strict convergence. Proc. R. Soc. Edinb. Sect. A 119 (1991), 265-278.

[7] S. Hencl, P. Koskela \& J. Onninen, Homeomorphisms of Bounded Variation, Arch. Ration. Mech. Anal. 186 (2007), no. 3, 351-360.

[8] S. Hencl \& A. Pratelli, Diffeomorphic Approximation of $W^{1,1}$ Planar Sobolev Homeomorphisms, to appear on J. Eur. Math. Soc. (2016)

[9] T. Iwaniec, L. V. Kovalev and J. Onninen, Diffeomorphic Approximation of Sobolev Homeomorphisms, Archive for Rational Mechanics and Analysis 201 (2011), no. 3, 1047-1067. 
[10] J. Kristensen \& F. Rindler, Characterization of generalized gradient Young measures generated by sequences in $W^{1,1}$ and BV, Arch. Ration. Mech. Anal. 197 (2010), no. 2, 539-598.

[11] J. Kristensen \& F. Rindler, Relaxation of signed integral functionals in BV, Calc. Var. Partial Differential Equations 37 (2010), no. 1-2, 29-62.

[12] C. Mora Corral \& A. Pratelli, Approximation of piecewise affine homeomorphisms by diffeomorphisms, J. Geom. Anal. 24 (2014), no. 3, 1398-1424.

[13] A. Pratelli, On the bi-Sobolev planar homeomorphisms and their approximation, Nonlinear Anal. 154 (2017), 258-268.

[14] A. Pratelli \& E. Radici, On the planar minimal BV extension problem, preprint (2017).

[15] E. Radici, A planar Sobolev extension theorem for piecewise linear homeomorphisms, Pacific J. Math., 283 (2016), no. 2, 405-418.

[16] F. Rindler \& G. Shaw, Strictly continuous extension of functionals with linear growth to the spaces BV, Q.J. Math. 66 (2015), no. 3, 953-978.

Department of Mathematics, University of Erlangen, Cauerstrasse 11, 90158 Erlangen, GerMANY

E-mail address: pratelli@math.fau.de

Department of Information Engineering, Computer Science and Mathematics, University of L'Aquila, Via Vetoio 1, 67100 Coppito (AQ), Italy

E-mail address: emanuela.radici@univaq.it 